\title{
A multi-domain approach for smoothed particle hydrodynamics simulations of highly complex flows
}

\author{
Alessandra Monteleone ${ }^{\mathrm{a}}$, Mauro De Marchis ${ }^{\mathrm{b}}$, Barbara Milici ${ }^{\mathrm{b}}$ \& Enrico \\ Napoli $^{\text {a* }}$ \\ a Dipartimento di Ingegneria Civile, Ambientale, Aerospaziale, dei Materiali, \\ Università degli Studi di Palermo, Viale delle Scienze, Palermo, Italy. E-mail: \\ enrico.napoli@unipa.it \\ b Facoltà di Ingegneria e Architettura, Università degli Studi di Enna "Kore", \\ Cittadella Universitaria,Enna, Italy.E-mail: mauro.demarchis@unikore.it
}

\begin{abstract}
An efficient and accurate method is proposed to solve the incompressible flow momentum and continuity equations in computational domains partitioned into subdomains in the framework of the smoothed particle hydrodynamics method. The procedure does not require any overlap of the subdomains, which would result in the increase of the computational effort. Perfectly matching solutions are obtained at the surfaces separating neighboring blocks. The block interfaces can be both planar and curved surfaces allowing to easily decompose even geometrically complex domains.

The smoothing length of the kernel function is maintained constant in each subdomain, while changing between blocks where a different resolution is required. Particles leaving each block through the interfaces are deactivated and correspondingly new particles are generated at the neighboring block using a dynamically adaptive procedure to control their frequency of release. No splitting and coalescing method is thus employed to take into account the different size and mass of the particles going through the interfaces. Mass conservation is guaranteed during the procedure, which is a challenging task in a Lagrangian method based on the domain decomposition.

The test cases in both 2D and 3D approximation show the accuracy of the method and its ability to strongly reduce the computational efforts through a multi-resolution approach.
\end{abstract}

Keywords: Smoothed particle hydrodynamics, ISPH, multi-block, domain decomposition, boundary conditions, mirror particles.

\footnotetext{
${ }^{*}$ Corresponding author
}

Preprint submitted to Computer Methods in Applied Mechanics and EngineeringApril 22, 2018 


\section{Introduction}

Smoothed particle hydrodynamics (SPH) is a Lagrangian particle method in which the equations are solved using discrete convolution integrals with filter functions of assigned shape, indicated as kernel functions. Each particle is assigned a support domain, including all the surrounding particles having distance lower than the product between the characteristic width $h$ of the kernel function (known as smoothing length) and a constant $k$ depending on the shape of the kernel function. The accuracy of the computation is directly related to the smoothing length, which plays a role corresponding to the cell dimension in grid-based methods. In order to obtain high quality solutions, a reasonably high number of particles must be contained in each support domain, maintaining a relatively regular space distribution during the time evolution of the simulation.

The number of particles $N$ depends on their isotropic initial distance $\Delta x$, which is commonly assigned as proportional to the smoothing length $h$. Thus, in 3D computations, it is $N \propto h^{3}$. Each particle moreover is assigned a given mass $m=\rho \Delta x^{3}$, where $\rho$ is the particle density.

In mesh methods, in order to reduce the computational efforts, grids are usually non uniform, being stretched and/or clustered close to external or internal boundaries and in regions of the computational domains with high gradients of the hydrodynamic variables. On the contrary, in the classic SPH approach the smoothing length is uniform in space due to the difficulty of changing the width of the kernel function while the particles move from one region to another. The computational efforts are thus very high, since the value of the smoothing length must be chosen according to the one imposed by the regions requiring the finest discretization. The same computational overload would be undergone by grid-based methods employing in the whole domain cubic cells with constant size.

In order to overcome this drawback, several refinement strategies have been proposed in the SPH approach, using a variable smoothing length $\left(h=h\left(\mathbf{x}_{\mathbf{p}}\right)\right.$, where $\mathbf{x}_{\mathrm{p}}$ is the particle position) to increase the computational efficiency (Feldman and Bonet, 2007; López et al., 2013). Due to the Lagrangian nature of the method, these approaches require introducing splitting and coalescing techniques for the particles, since their dimension and support domain is required to adapt to the space dependent $h$ (Vacondio et al., 2013a,b; Xiong et al., 2013; Spreng et al., 2014; Barcarolo et al., 2014; Vacondio et al., 2016; Hu et al., 2017).

Here a different approach is proposed, based on multi-domain decomposition, which allows to use different values of the smoothing length in the blocks, while maintaining inside each of them the simplicity of the classic SPH method with constant $h$. In the proposed procedure, differently from Shibata et al. (2017), no overlapping of the subdomains is employed, thus avoiding any artificial increase of the computational domain. During the simulations the particles leaving each subdomain through the internal interfaces (outflow) are removed from the computation, while a specific procedure allows to generate new particles in correspondence of inflow interfaces. The procedure, which is similar to the one employed by Napoli et al. (2016) in the coupling of the SPH and finite 
volume methods, guarantees perfect mass conservation in each block and in the whole domain and allows each interface to switch from inflow to outflow (and viceversa) during the simulation. Inflow and outflow conditions are allowed to coexist in the same interface, as discussed by Monteleone et al. (2017).

The proposed multi-domain technique is employed here in the framework of the Incompressible SPH approach (Lind et al., 2012; Napoli et al., 2015; Kunz et al., 2016), based on the fractional-step resolution of the Navier-Stokes equations. The discretized Pressure Poisson equations, required to impose the nulldivergence condition, are solved using for the whole domain a unique equation system, obtained putting together the single sub-systems of each subdomain. The solution of the system is obtained through a pre-conditioned BicGStab algorithm (Van der Vorst, 1992). The simultaneous solution of the sub-systems allows to obtain a perfectly matched solution among the single subdomains, where different particle sizes are employed.

Three different test cases are used to show the efficiency and accuracy of the proposed method: the 3D unsteady channel flow in a cylindrical pipe, the 2D vortex shedding in the wake of a circular cylinder and the hemodynamic systolic cycle in a cerebral vessel with an aneurysmatic sac. Although all the test cases are relative to confined flows, in principle no limitation exists to the application of the proposed method to free-surface flows.

The paper is organized as follows: in the second section a general description of the ISPH method is given, in the third the proposed multi-domain technique is described in detail and in the fourth the validation through the employed test cases is discussed. Some conclusions are finally drawn in the last section.

\section{ISPH basic formulation}

The generic function $f$ at the position $\mathbf{x}$ can be expressed in the smoothed form $\langle f(\mathbf{x})\rangle$ through the integral convolution:

$$
\langle f(\mathbf{x})\rangle=\int_{D} f(\mathbf{x}) W\left(\mathbf{x}-\mathbf{x}^{\prime}, h\right) d \mathbf{x}^{\prime}
$$

where $W$ is a kernel function of smoothing length $h$ and $D$ is the computational domain.

In the SPH approximation the fluid is represented by a finite number of particles which move according to the Navier-Stokes equations. Each $i$ particle has its own mass and density $\left(m_{i}\right.$ and $\left.\rho_{i}\right)$ and support domain $\Omega_{i}$ whose dimension depends on the smoothing length $h$. The convolution integral (1) for the $i$ particle can be thus approximated as the summation over the $N_{i}$ particles lying into $\Omega_{i}$ :

$$
f_{i}=\sum_{j=1}^{N_{i}} \frac{m_{j}}{\rho_{j}} f_{j} W_{i j}
$$

where the index $j$ is used to indicate the properties of the generic $j$ particle in $\Omega_{i}$ and $W_{i j}=W\left(\mathbf{x}_{i}-\mathbf{x}_{j}, h\right)$. 


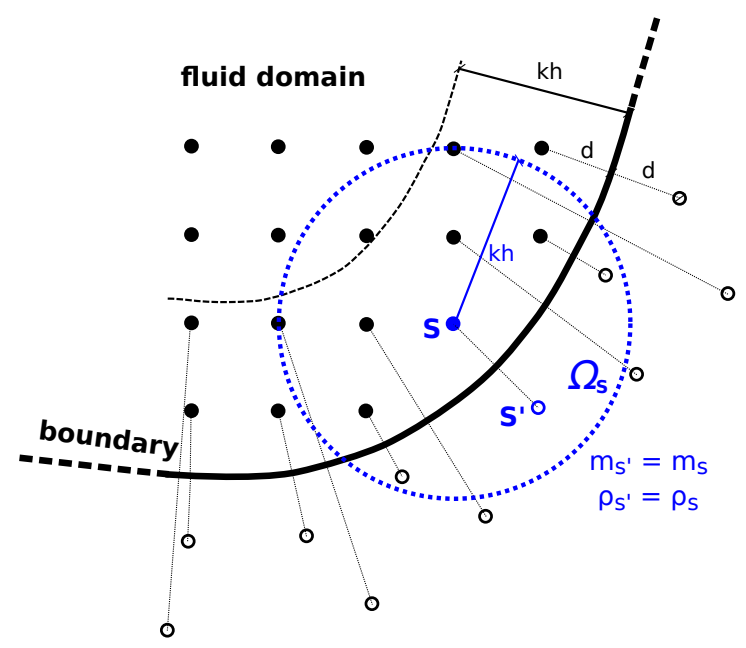

Figure 1: Sketch of the generation of the mirror particles (empty black circles) from effective particles (full black circles) having distance $d<k h$ from the boundary (bold black line). The dotted blue line around the effective particle $S$ indicates its support domain $\Omega_{S}$.

As discussed by Liu and Liu (2010) and Morris et al. (1997), the first derivatives and the Laplacian operator can be expressed as

$$
\left.\frac{\partial f}{\partial x}\right|_{i}=-\sum_{j=1}^{N_{i}} \frac{m_{j}}{\rho_{j}}\left(f_{i}-f_{j}\right) \frac{\partial W_{i j}}{\partial x}
$$

and

$$
\nabla^{2} f_{i}=\sum_{j=1}^{N_{i}} 2 \frac{m_{j}}{\rho_{j}} \frac{\left(\mathbf{x}_{i}-\mathbf{x}_{j}\right) \cdot \nabla W_{i j}}{d_{i j}^{2}}\left(f_{i}-f_{j}\right)
$$

where the symbol "." indicates the scalar product, $\nabla W_{i j}$ is the gradient of the kernel function and $d_{i j}$ is the distance between the $i$ and $j$ particles.

In order to account for the truncation of the support domain occurring near the boundaries of the domain, in SPH mirror particles are usually displaced outside the computational domain, having mass and density equal to the effective particles from which they are generated (Fig. 1). The specific boundary conditions (no-slip, free-slip, Dirichlet, etc.) are imposed assigning to the mirror particles values coherent with the selected condition. An extended discussion on the mirror particle generation and suitable assignment of boundary conditions can be found in Napoli et al. (2015).

In the ISPH approach the Navier-Stokes equations are solved in three steps. First the non-solenoidal intermediate velocity $\mathbf{u}_{i}^{*}$ is obtained for each $i$ particle $(i=1, \ldots N)$ solving the equations after having removed the pressure gradient (predictor-step). In the SPH approximation the resulting equations read as: 


$$
\begin{aligned}
\frac{\mathbf{u}_{i}^{*}-\mathbf{u}_{i}^{r}}{\Delta t} & =\nu_{i}\left[\frac{3}{2} \sum_{j=1}^{N_{i}} 2 \frac{m_{j}}{\rho_{j}} \frac{\left(\mathbf{x}_{i}^{r}-\mathbf{x}_{j}^{r}\right) \cdot \nabla W_{i j}}{d_{i j}^{2}}\left(\mathbf{u}_{i}^{r}-\mathbf{u}_{j}^{r}\right)+\right. \\
& \left.-\frac{1}{2} \sum_{j=1}^{N_{i}} 2 \frac{m_{j}}{\rho_{j}} \frac{\left(\mathbf{x}_{i}^{r-1}-\mathbf{x}_{j}^{r-1}\right) \cdot \nabla W_{i j}}{d_{i j}^{2}}\left(\mathbf{u}_{i}^{r-1}-\mathbf{u}_{j}^{r-1}\right)\right]+\mathbf{g}
\end{aligned}
$$

where boldface symbols are used to indicate vectors, $\mathbf{u}_{i}$ is the particle velocity, $\Delta t$ is the time step, $\nu_{i}$ is the particle kinematic viscosity, $\mathbf{g}$ is the mass force and the superscripts $r$ and $r-1$ are used to indicate time levels. The AdamsBashfort scheme is used in eqn. (5) to obtain a second-order accurate explicit approximation.

In order to obtain the corrective velocity field, the Pressure Poisson equations are then solved for each particle:

$$
\sum_{j=1}^{N_{i}} 2 \frac{m_{j}}{\rho_{j}} \frac{\left(\mathbf{x}_{i}^{r}-\mathbf{x}_{j}^{r}\right) \cdot \nabla W_{i j}}{d_{i j}^{2}}\left(\psi_{i}-\psi_{j}\right)=\frac{1}{\Delta t} \sum_{j=1}^{N_{i}} \frac{m_{j}}{\rho_{j}}\left(\mathbf{u}_{i}^{*}-\mathbf{u}_{j}^{*}\right) \cdot \nabla W_{i j}
$$

where $\psi$ is the pseudo-pressure having the dimension of the kinematic pressure $(p / \rho)$ and the right-hand side term is the opposite of the divergence of the intermediate velocity divided by the time step. The boundary conditions for eqn. (6) are obtained assigning to the mirror particles the Neumann condition:

$$
\left.\frac{\partial \psi}{\partial n}\right|_{i}=\frac{u_{i n}^{r+1}-u_{i n}^{*}}{\Delta t}
$$

where the velocities are calculated at the intersection point between the boundary and the normal line starting from $i, n$ is the direction normal to the boundary at the same intersection point and the subscript $n$ indicates normal components $\left(u_{i n}=\mathbf{u}_{\mathbf{i}} \cdot \mathbf{n}\right)$. The boundary conditions for the intermediate velocities $\mathbf{u}_{i}^{*}$ are obtained through an extrapolation from the interior values as discussed by Zang et al. (1994) in the framework of the finite-volume application of the fractionalstep method.

In the third step the updated solenoidal velocity is finally obtained as:

$$
\mathbf{u}_{i}^{n+1}=\mathbf{u}_{i}^{*}-\Delta t \sum_{j=1}^{N_{i}} \frac{m_{j}}{\rho_{j}}\left(\psi_{i}-\psi_{j}\right) \nabla W_{i j}
$$

\section{The proposed multi-domain SPH method}

\subsection{The multi-domain decomposition}

As discussed in the Introduction, in order to adapt the numerical method to the spatial resolution required in each subregion, the computational domain 


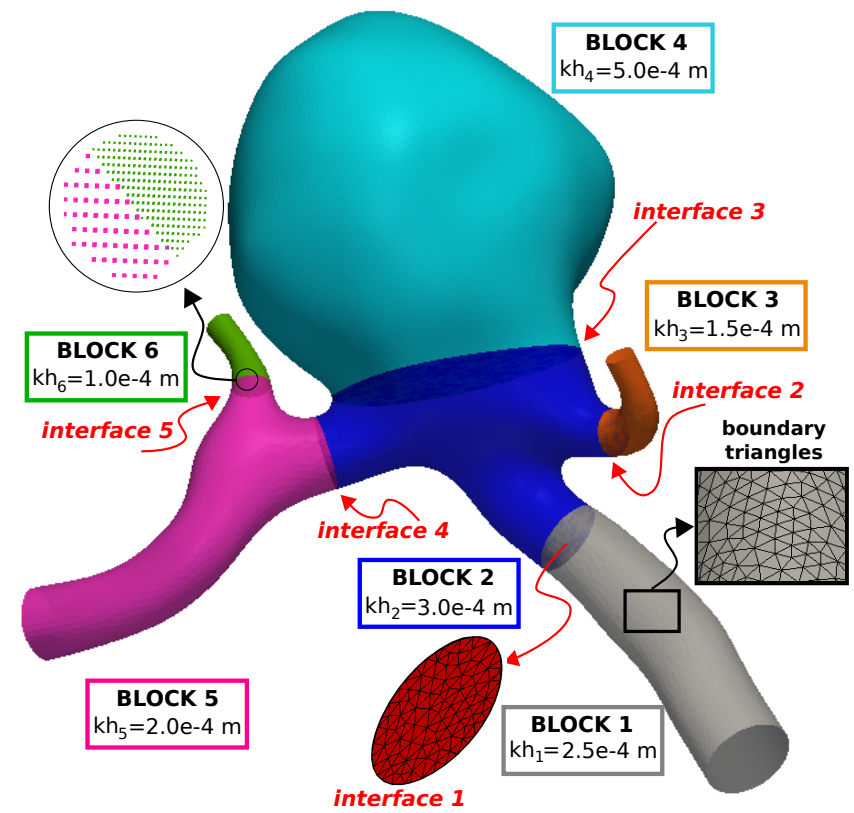

Figure 2: Sketch of the subdivision into 6 blocks of the computational domain analyzed in Test Case 3 (subsection 4.3). The external surfaces and the block interfaces are discretized into triangles (e.g., the rectangular gray area in block 1 and the elliptical red area at the interface between blocks 1 and 2). The change in the particle initial distance is visible in the enlargement inside the circular black line in the vicinity of the interface between blocks 5 and 6.

is partitioned in non-overlapping blocks (Fig. 2, where a scheme relative to the test case in Section 4.3 is shown). The blocks are separated by plane or curved surfaces which will be indicated in the following as block interfaces (or simply interfaces). These separation surfaces can be discretized into triangles, which allow to obtain suitable descriptions of complex shapes while easily identifying the normal directions (Napoli et al., 2015).

The smoothing length $h$ and the relative initial particle distance $\Delta x$ are maintained constant inside each block, while different values are assigned to the particles contained in different blocks. As a consequence, the classic SPH equations (eqns. (5)-(8) in the proposed approach) can be used inside each block, although specific procedures must be included to account for the proper treatment of near-interface regions. Specifically, since in these areas the support domain of the particles can be truncated by the block interfaces, additional interface particles (IP in the following) are added in the neighboring subdomain. The IP particles play an important role in order to obtain a suitable matching of the solution in neighboring blocks, as will be discussed in the next subsection 3.2 .

The IP particles are generated from the effective particles having distance shorter than $\Delta x$ from one of the interfaces. The particles are displaced in 
the direction normal to the interface, at distance from the relative generating particle equal to $v \Delta x$ with $v=1,2, \ldots V$ where $V$ is the integer part of the ratio $(k h / \Delta x)$. The number of interface particles generated from each effective particle is thus selected to reach the contour of the support domain.

In Fig. 3.a IP generation from the effective particles of block $A$ is described. For the sake of clarity a bi-dimensional sketch is considered in the figure, where the triangle interfaces are represented by a segment (bold red line in the figure). The particle $S$ of block $A$, having distance from the interface shorter than $\Delta x_{A}$, generates two $I P$ particles in the direction normal to the interface $\left(S^{\prime}\right.$ and $\left.S^{\prime \prime}\right)$. The two $I P$ particles $S^{\prime}$ and $S^{\prime \prime}$ have distance from the generating particle $S$ equal to $\Delta x_{A}$ and $2 \Delta x_{A}$, respectively. These particles are contained in the neighboring block $B$. A $3 D$ scheme is shown in Fig. 3.b where the interface is a curved surface. In the figure the generation is described considering two effective particles of the blocks $A$ and $B$ ( $S$ and $R$ in the figure, respectively). Since $S$ and $R$ have distance from the relative triangle planes $(d R$ and $d S)$ shorter than $\Delta x_{B}$ and $\Delta x_{A}$, respectively, two IP particles are generated from each of them. The lines normal to the triangle planes are identified, allowing to generate the $I P$ particles $R^{\prime}$ and $R^{\prime \prime}$ and the $I P$ particles $S^{\prime}$ and $S^{\prime \prime}$ having distance from the corresponding effective particles equal to once and twice the starting particle distances $\left(\Delta x_{A}\right.$, for block $A$ and $\Delta x_{B}$ for block $\left.B\right)$.

As discussed in the Introduction, in the procedure no overlapping region is created between neighboring subdomains, which are entirely separated. Nevertheless, since the effective particles of a block generate, through the interface, $I P$ particles lying in the neighboring subdomain, an overlapping region is created where effective particles of a block coexist with IP particles generated by the neighboring block. In Fig. 4, relative to the first test case discussed in subsection 4.1, two subdomains $A$ (green effective particles) and $B$ (blue effective particles) are shown. The effective particles of $A$ and $B$ are separated by an interface which is represented by red triangles in Fig. 4.a (3D view) and is plotted as a red line in Fig. 4.b (2D view). No effective particles of block $B$ (blue points in the figure) are contained in block $A$ (which is filled with green particles) and viceversa, since the blocks are separated. On the contrary, the $I P$ particles generated by the effective particles of block $B$ (red points in the figure) are contained inside block $A$, while on the other hand the $I P$ particles generated by block $A$ (black points) are contained inside block $B$. As it will be discussed in section 3.2, the hydrodynamic values of the $I P$ particles generated by a block are obtained through an interpolation starting from the effective particles of the block in which they are contained.

\subsection{The solution matching at the block interfaces}

To obtain the SPH approximations of eqns. (2)-(4) at the effective particles close to the block interfaces, the values of the $f$ variables at the IP particles must be identified.

As shown in Fig. 5, the $I P$ particles of block $A$ neighboring block $B$ are contained inside $B$. Their hydrodynamic properties can be thus obtained through a Taylor series expansion around the closest effective particle of block $B$. Using 


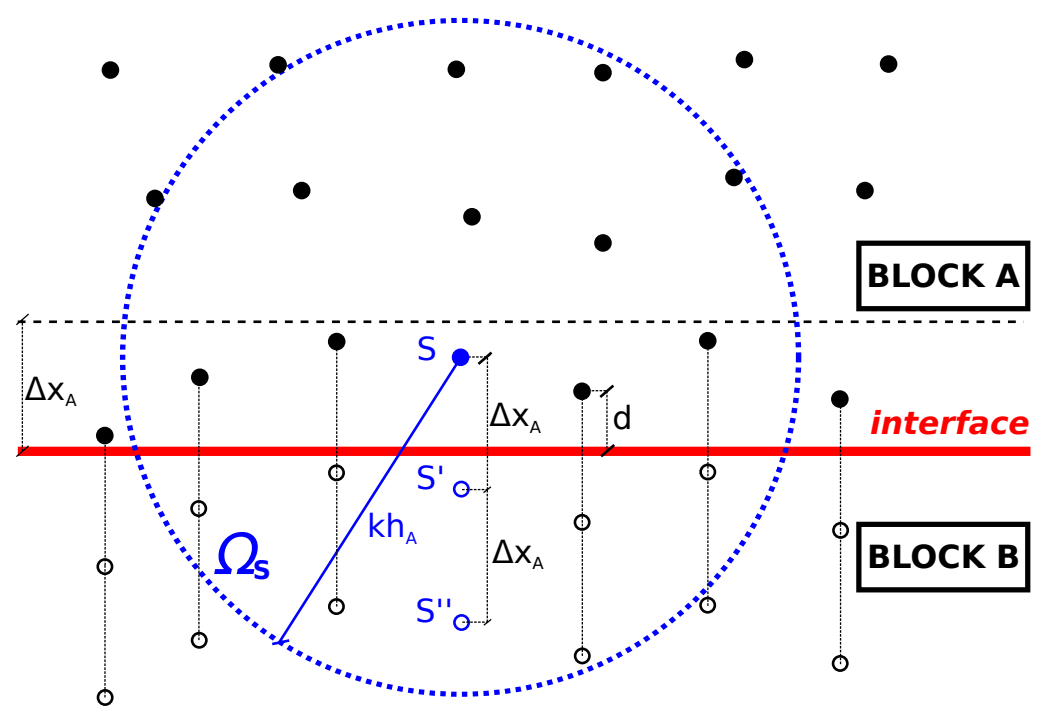

(a)

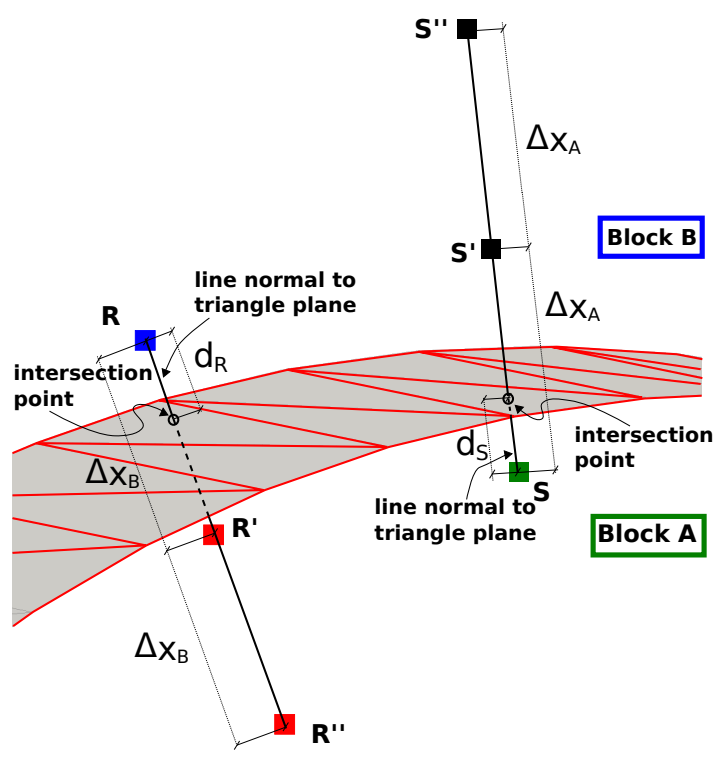

(b)

Figure 3: Sketch of the IP particle generation. a) $2 D$ scheme where the effective particles of block $A$ (full black circles) having distance $d<\Delta x_{A}$ from the block interface (bold red line) generate $I P$ particles (empty black circles). The dotted blue circle around the effective particle $S$ indicates the support domain $S$, containing the particles $S^{\prime}$ and $S^{\prime \prime}$; b) $3 D$ scheme, where a curved interface surface is used. Effective $R$ and $S$ particles generate two $I P$ particles each in the neighboring subdomain in the direction normal to the interface triangle. 


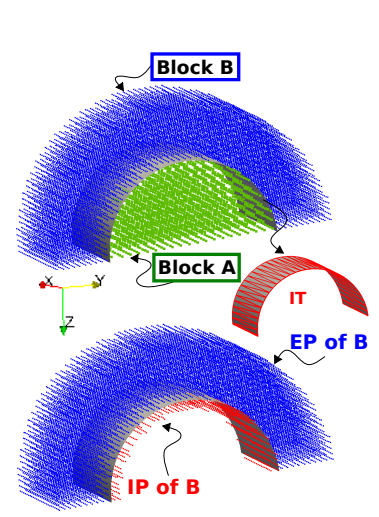

(a)

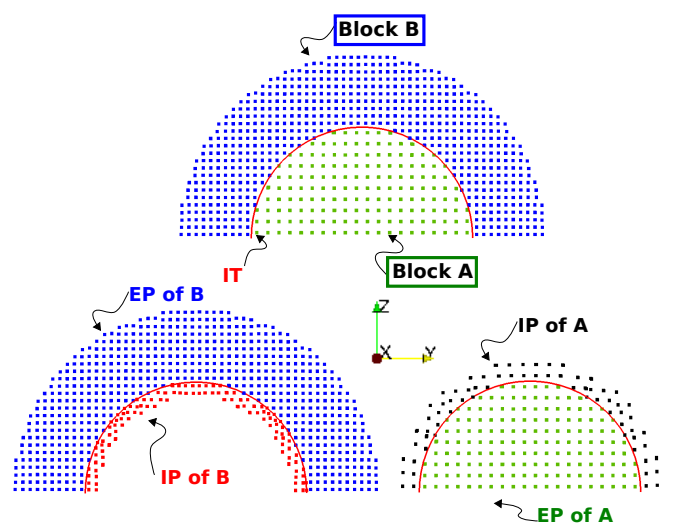

(b)

Figure 4: $E P$ : effective particles; IP: interface particles; IT: interface triangles; Block $A$ : green particles; Block $B$ : blue particles. Block subdivision. a) $3 D$ sketch; b) $2 D$ sketch.

the symbols $P$ and $R$ to indicate an $I P$ particle of block $A$ and the closest effective particle of block $B$, respectively, the expansion reads as:

$$
f_{P}=f_{R}+\left[\sum_{j=1}^{N_{R}} \frac{m_{j}}{\rho_{j}}\left(f_{j}-f_{R}\right) \nabla W_{R j}\right] \cdot\left(\mathbf{x}_{\mathbf{P}}-\mathbf{x}_{\mathbf{R}}\right)
$$

where the sum is extended to the $N_{R}$ particles inside the $R$ support domain $\Omega_{R}$ with radius $k h_{B}$ (dotted green line) and the expansion is truncated at first order.

As it can be seen in the figure, the support domain $\Omega_{R}$ contains both effective and $I P$ particles (full and empty squares in the figure, respectively) and thus eqn. (9) can be rewritten as:

$f_{P}=f_{R}+\left[\sum_{e=1}^{N_{R, e f f}} \frac{m_{e}}{\rho_{e}}\left(f_{e}-f_{R}\right) \nabla W_{R e}+\sum_{g=1}^{N_{R, I P}} \frac{m_{g}}{\rho_{g}}\left(f_{g}-f_{R}\right) \nabla W_{R i}\right] \cdot\left(\mathbf{x}_{\mathbf{P}}-\mathbf{x}_{\mathbf{R}}\right)$

using the subscripts $e$ and $g$ to separate the summations over the $N_{R, \text { eff }}$ effective particles and the $N_{R, I P} I P$ ones (with $N_{R}=N_{R, \text { eff }}+N_{R, I P}$ ). This separation is useful since the values $f_{g}$ in eqn. (10) are unknowns, which can be obtained using corresponding Taylor series expansions around the closest effective particles of block $A$.

As a consequence, the eqns. (10) relative to the neighboring $A$ and $B$ blocks must be solved as a system containing one equation for each $I P$ particle of the blocks: 


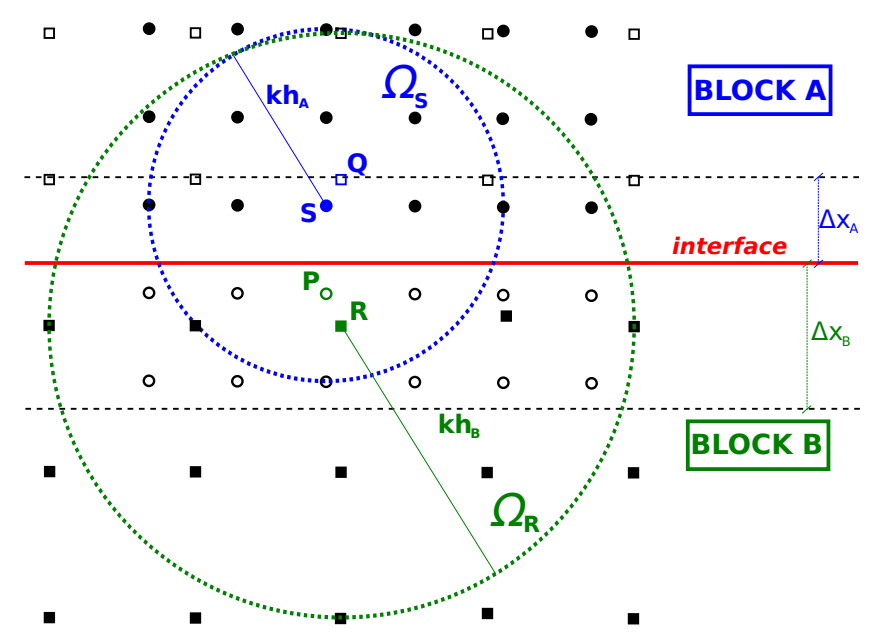

Figure 5: Sketch of the interface particle distribution. Full and empty black circles: effective and $I P$ particles of block $A$; full and empty black squares: effective and IP particles of block $B$; bold red line: block interface; dotted blue and green lines: support domains $\Omega_{S}$ and $\Omega_{R}$ of particles $S$ and $R$ belonging to block $A$ and $B$, respectively.

$$
\begin{array}{cc}
f_{P}^{A}-\sum_{g=1}^{N_{R, I P}} c_{g} f_{g}^{B}=R H S_{P} & P=1, \cdots N_{I P}^{A} \\
f_{Q}^{B}-\sum_{g=1}^{N_{S, I P}} d_{g} f_{g}^{A}=R H S_{Q} & Q=1, \cdots N_{I P}^{B}
\end{array}
$$

where $Q$ is an $I P$ particle of block $B$ whose closest effective particle in block $A$ is $S$, the superscripts $A$ and $B$ are used to indicate particles of blocks $A$ and $B$, respectively, $N_{I P}^{A}$ and $N_{I P}^{B}$ are the numbers of $I P$ particles in the blocks, the coefficients $c_{g}$ and $d_{g}$ are

$$
\begin{aligned}
c_{g} & =\frac{m_{g}}{\rho_{g}} \nabla W_{R g} \cdot\left(\mathbf{x}_{\mathbf{P}}-\mathbf{x}_{\mathbf{R}}\right) \\
d_{g} & =\frac{m_{g}}{\rho_{g}} \nabla W_{S g} \cdot\left(\mathbf{x}_{\mathbf{Q}}-\mathbf{x}_{\mathbf{S}}\right)
\end{aligned}
$$

and the right-hand-side terms $R H S_{P}$ and $R H S_{Q}$ are 


$$
\begin{gathered}
R H S_{P}=f_{R}^{B}+\left[\sum_{e=1}^{N_{R, e f f}} \frac{m_{e}}{\rho_{e}}\left(f_{e}^{B}-f_{R}^{B}\right) \nabla W_{R e}-\sum_{g=1}^{N_{R, I P}} \frac{m_{g}}{\rho_{g}} f_{R}^{B} \nabla W_{R g}\right] \cdot\left(\mathbf{x}_{\mathbf{P}}-\mathbf{x}_{\mathbf{R}}\right) \\
R H S_{Q}=f_{S}^{A}+\left[\sum_{e=1}^{N_{S, e f f}} \frac{m_{e}}{\rho_{e}}\left(f_{e}^{A}-f_{S}^{A}\right) \nabla W_{S e}-\sum_{g=1}^{N_{S, I P}} \frac{m_{g}}{\rho_{g}} f_{S}^{A} \nabla W_{S g}\right] \cdot\left(\mathbf{x}_{\mathbf{S}}-\mathbf{x}_{\mathbf{Q}}\right)
\end{gathered}
$$

The equation system (11) must be solved at each interface. In order to obtain vectorial values (e.g., the velocities $\mathbf{u}$ ), the system must be solved at each interface once for each component, using the same coefficient matrix and updating the right-hand-side only.

A different procedure is used for the pseudo-pressure $\psi$, since eqns. (9) must be solved simultaneously with the Pressure Poisson eqns. (6). This implies that all the $f_{R}$ and $f_{j}$ terms in eqn. (9) are unknowns and, moreover, the equations relative to the different interfaces must be solved simultaneously too. As a result, a global system made of the $N$ Pressure Poisson equations and the $N_{I P}$ interface particle Taylor expansions must be solved, where $N$ and $N_{I P}$ are the sums of the effective and interface particles belonging to each block.

\subsection{The inflow/outflow procedure through the block interfaces}

The employment of a multi-domain approach in the framework of a Lagrangian method, as SPH, requires taking into account the movements of the particles from one block to another.

In the proposed approach the inflow and outflow procedures must be handled separately. Specifically, at the end of each time step, after having calculated the particle velocities through eqn. (8) and having accordingly updated their position, it is checked for each particle if it has gone through one of the interfaces. In this case the interface is considered an outflow for the block and the particle is simply removed from the list of particles of the block it comes from. External boundaries and internal interfaces connecting neighboring blocks are treated in the same way from this point of view, since in both cases the particle is leaving the block to which it belongs. As it is shown in Fig. 6.a, thus, a particle $P$ leaving block $A$ towards $B$ is cancelled at the next time step $(r+1$ in the figure). If at the end of the time step a particle approaches one of the interfaces without crossing it (particle $Q$ in the figure), one or more $I P$ particles are generated as it has been described in section 3.1.

On the other hand, considering the scheme in Fig. 6.b, the entering of new particles in block $B$ is handled as described in the following. At the end of each time step the region between the starting particle distance $\Delta x$ and $k h$ from the interfaces is considered (light green area in the figure). In order to verify if the generation of new particles is required into this region, for each effective particle contained in this checking area ( $S$ and $T$ in the figure) and having positive velocity component in the direction normal to the interface (thus pointing towards the interior of the block), a conical scan region is identified, 


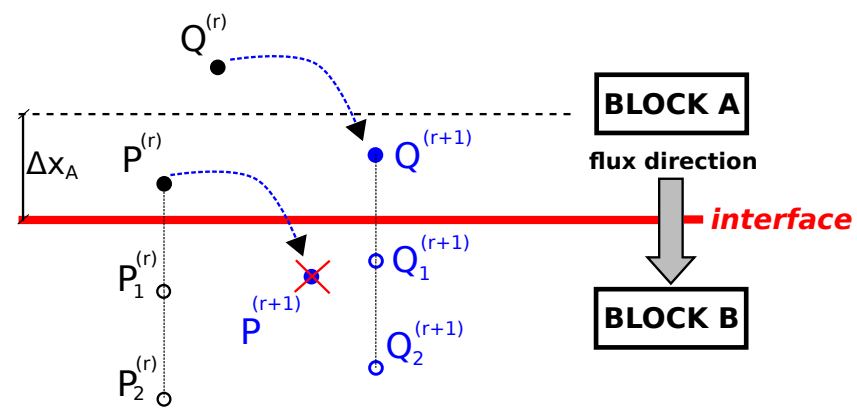

(a)

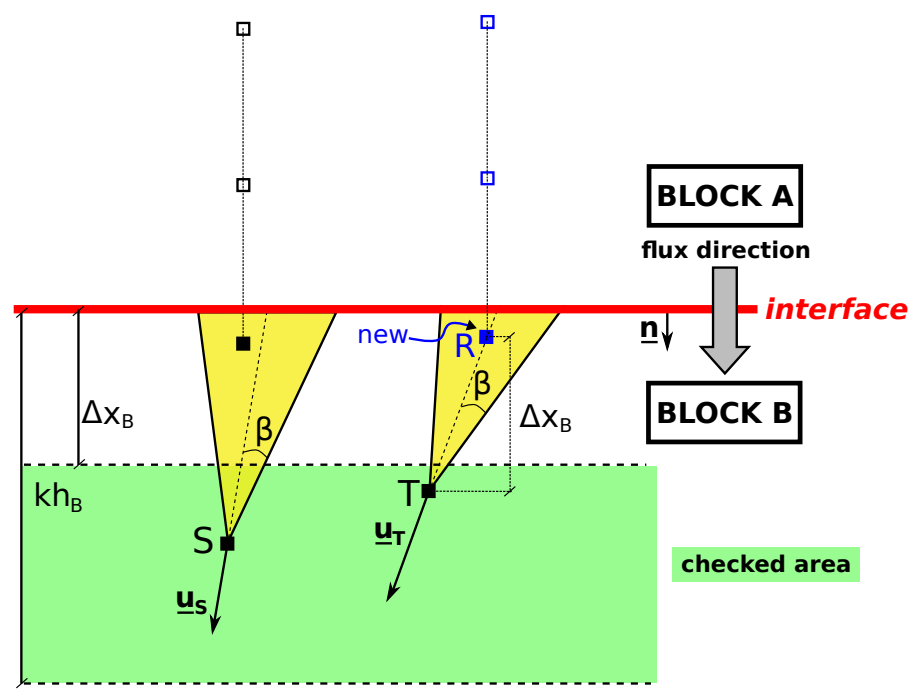

(b)

Figure 6: Inflow/outflow procedure sketch. a) Outflow. Full and empty black circles: effective and $I P$ particles of block $A$ at time $r$; full and empty blue circles: effective and $I P$ particles of block $A$ at time $r+1$; b) Inflow. Full and empty black squares: effective and $I P$ particles of block $B$; yellow area: conical scan region with opening angle $\beta ; R$ : effective particle generated inside the cone with vertex in $T$; empty blue squares: $I P$ particles of $R$; green area: checked region. 
which is indicated with the yellow color in the $2 D$ scheme shown in the figure. The vertex of the cone and the axis direction are assigned equal to the particle position and velocity direction, respectively, as shown in Fig. 6.b (particles $S$ and $T$ ). The conical volume thus is placed on the upstream region contained between the checked particle and the interface. The region is used to verify if the movement of the considered particle towards the interior of the block is causing the development of an empty region, which would require the generation of a new particle to fill it. Thus, a new particle is released if no effective particle is found in the conical region, as occurring in the figure with reference to the cone with vertex in $T$. The new particle ( $R$ in the figure) is displaced at distance $\Delta x$ from the cone vertex along the cone axis direction. If on the contrary some effective particles are found in the conical region (which occurs in the figure for the cone with vertex in $S$ ), no particle is generated inside the cone since no empty region has been identified.

In order to control the frequency of new particle release, the cone amplitude $\beta$ is dynamically adjusted at each time step: specifically, the angle $\beta$ is increased by a fixed amount $\left(1^{\circ}\right.$ in our test cases, starting from the initial value of $\left.30^{\circ}\right)$ when the total number of effective particles in the block becomes higher than the starting number $N_{0}$ and it is reduced by the same amount in the opposite case. The dynamic updating of the cone amplitude allows to maintain constant the number of particles in each block, thus correctly satisfying the continuity constraint, since the widening of the cone increases the probability to find effective particles inside the conical region and thus reduces the frequency of release. The efficiency of the procedure, which is similar to the one employed by Napoli et al. (2016) in a FVM-SPH coupled approach, will be demonstrated in the test cases discussed in the next section. The particles removed from the computation after having left a block through the interface are saved in a storage list, from which they are collected when new particles have to be released. The continuous increase of the existing particles is thus avoided, which would occur if the released particles would be newly generated instead of been taken from the list of the previously cancelled ones.

To summarize, the inflow/outflow procedure is applied as follows:

- Deleted particles. If one effective particle at the end of a time step crosses one of the triangles defining an external boundary or an internal interface (connecting one block with the neighboring one) it will be removed from the computation. The interface triangle is thus considered an outflow for the current block;

- Particles with distance $d<\Delta x$ from the interface. If an effective particle has distance $d$ from the interface shorter than the particle distance $\Delta x$, two or more $I P$ particles are generated as discussed in section 3.1. This is valid for both inflow and outflow interfaces;

- Particles with $\Delta x<d<k h$ and $\mathbf{u}_{i} \cdot \mathbf{n}>0$. If an effective particle has distance from the interface ranging between the starting particle distance $\Delta x$ 
and $k h$, and the velocity component normal to the interface plane is positive $\left(u_{i} \cdot n>0\right.$, implying an inflow condition) it must be checked if the release of a new particle is required. Thus the procedure described above based on the cone region analysis starts;

- Particles with $\Delta x<d<k h$ and $\mathbf{u}_{i} \cdot \mathbf{n}<0$. If an effective particle has distance from the interface ranging between $\Delta x$ and $k h$ but its velocity component normal to the interface triangle is negative (thus the particle points outside the interior of the particle block), no new particle is released starting from the current effective particle, nor any IP particle is generated since the distance from the interface is larger than $\Delta x$.

\subsection{Overall summary of the proposed method}

In order to provide a general description of the single steps required to advance in time the solution in the proposed multi-domain technique, a flow chart is shown in Fig. 7. Although the method is applied here in the ISPH framework, it could be straightforwardly integrated in the WCSPH approach as well. The actions indicated in the flow chart are briefly explained in the following:

- ACTION 1: The domain is partitioned into non-overlapping blocks as explained in section 3.1. Each block has a different smoothing length and is separated from the neighboring ones by plane or curved interfaces. The action is a preparatory step, which is performed only once before starting the iterative process for the time marching of the solution.

- ACTION 2: For each block the mirror and IP particles are generated as discussed in sections 2 and 3.1 (see Figs. 1 and 3), respectively. The generation of $I P$ particles is performed considering the interfaces between blocks as if they were external boundaries.

- ACTION 3: In the predictor step, eqn. (5) is solved for the effective particles of each block to calculate the intermediate velocity $\mathbf{u}^{*}$.

- ACTION 4: The equation system (11) is solved at each interface to obtain the intermediate velocity $\mathbf{u}^{*}$ of the $I P$ particles generated from the neighboring blocks.

- ACTION 5: The pseudo-pressure $\psi$ of the effective and IP particles of all the blocks is calculated solving one single system made up of one Pressure Poisson equation (eqn. 6) for each effective particle and one interpolation equation (eqn. 9) for each IP particle.

- ACTION 6: In the corrector-step, the updated velocity $\mathbf{u}$ is calculated for each effective particle solving eqn. (8).

- ACTION 7: Identical to Action 4, considering the updated velocity $\mathbf{u}$. 
- ACTION 8: The position of the effective particles is updated using the corrected velocities $\mathbf{u}$ calculated in Action 6 .

- ACTION 9: As discussed in section 3.3, the particles crossing external outflow boundaries or internal block interfaces are deactivated and saved in a storage list.

- ACTION 10: New particles are released from inflow and interface triangles as described in section 3.3.

- ACTION 11: The mirror and IP particles are generated as in Action 2. This step is required here in order to perform the next action.

- ACTION 12: The shifting procedure proposed by Xu et al. (2009) is used to overcome the well-known tensile instability problem and improve the particle distribution.

After the twelfth action, the solution time is advanced by one time step and the procedure is restarted with Action 2. The activities specifically required by the proposed multi-domain technique are indicated in red in the flow chart of Fig. 7.

\section{Test cases}

The proposed procedure has been verified considering three test cases, both in $2 \mathrm{D}$ and $3 \mathrm{D}$ approximation, in the laminar regime.

In all the test cases the Wendland kernel function (Wendland, 1995) has been employed, with the value $k=2$ for the proportionality constant between the radius of the support domain and the smoothing length $h$. The starting particle distance $\Delta x$ has been set equal to $k h / 2$. In order to improve the particle distribution and to overcome the so called tensile instability related to the particle clustering along the streamlines (Monaghan, 2000), the shifting procedure proposed by Xu et al. (2009) has been used.

The equation systems (11) at each interface as well as the larger system of the Pressure Poisson equations have been solved using the BiCGStab method (Van der Vorst, 1992), with a pre-conditioning algorithm to speed-up the convergence (Saad, 2003).

The numerical model has been implemented in the open-source PANORMUS package (Napoli, 2011).

\subsection{Test case 1: Transient Poiseuille flow}

The first test case is the Poiseuille flow inside a circular pipe, which has been solved starting from the rest till achieving the steady-state. The pipe diameter has been set to $D=0.1 \mathrm{~m}$, with length $L=D$. The flow density and kinematic viscosity have been set to $\rho=1000 \mathrm{~kg} / \mathrm{m}^{3}$ and $\nu=1 \cdot 10^{-6} \mathrm{~m}^{2} / \mathrm{s}$ and the pressure gradient $\nabla P=2 \cdot 10^{-2} \mathrm{~Pa} / \mathrm{m}$ has been used to drive the flow. The resulting analytical maximum velocity is $u_{\max }=0.0125 \mathrm{~m} / \mathrm{s}$, with a Reynolds 


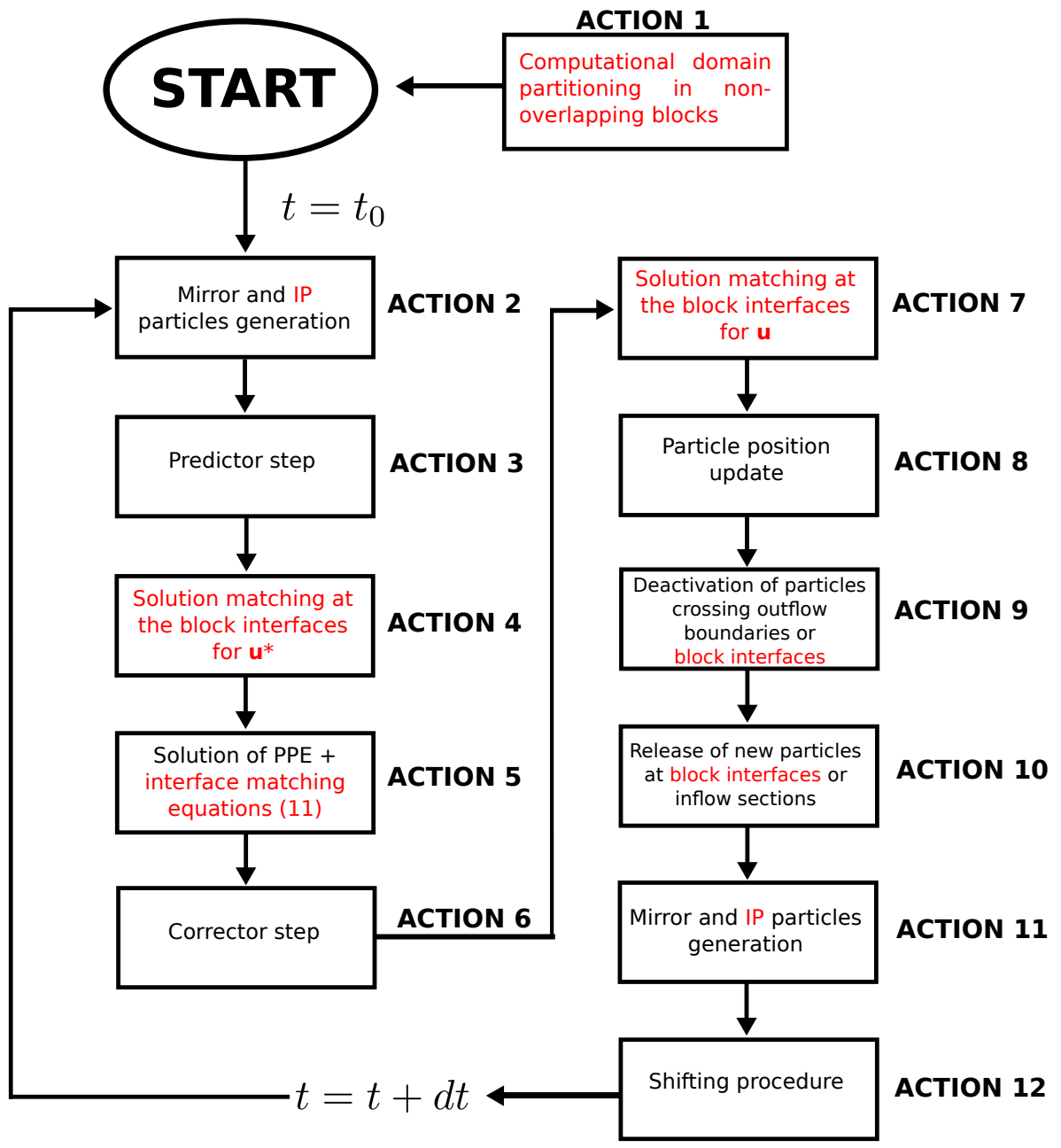

Figure 7: Flow chart of the proposed method in the ISPH framework. The actions closely related to the multi-domain approach are highlighted with the red color. 

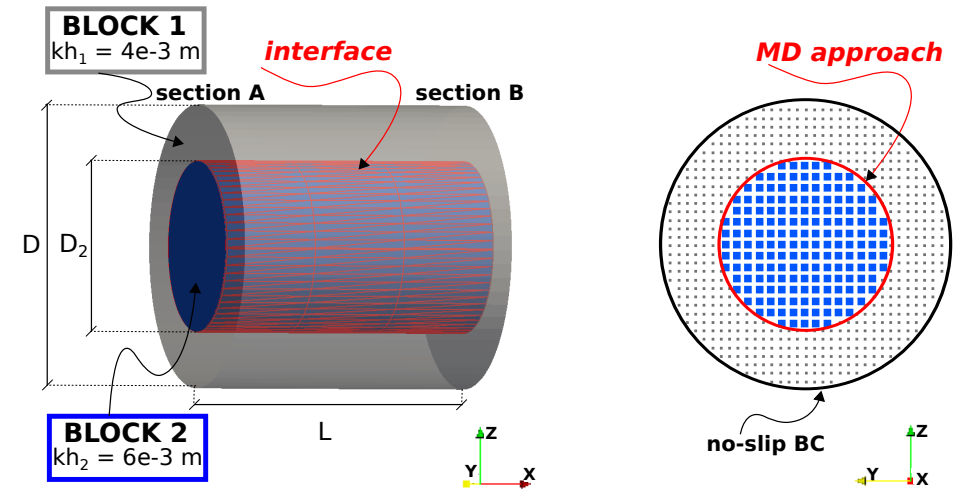

Figure 8: Test case 1. Domain subdivision into blocks 1 (gray) and 2 (blue). The bold red line indicates block interface triangles, while $A$ and $B$ are the inflow and outflow sections, respectively. a) surface representation; b) cross-section with particle representation.

number $R e=u_{m} D / \nu=625$, where $u_{m}$ is the cross-section averaged streamwise velocity at the steady-state.

The domain has been partitioned into two coaxial cylindrical blocks, as shown in Fig. 8, with the diameter of the internal block $D_{2}=0.6 D$. The interface (plotted in red color in the figure) is therefore a curved surface corresponding to the internal lateral wall of block 1 and the external lateral wall of block 2. The smoothing lengths of the external and internal blocks have been set to $h_{1}=2 \cdot 10^{-3} \mathrm{~m}$ and $h_{2}=3 \cdot 10^{-3} \mathrm{~m}$, respectively (correspondent to $k h_{1}=4 \cdot 10^{-3} \mathrm{~m}$ and $k h_{2}=6 \cdot 10^{-3} \mathrm{~m}$ ). The resulting initial number of effective particles is equal to $N 0_{1}=62750$ in the first block and $N 0_{2}=10428$ in the second one. The reduction of the particle numbers with respect to the value obtained using the smallest value of $k h$ in the whole domain is quite moderate in this simple test case (about $25 \%$ only) since the geometry and parameters have been chosen only to show the accuracy of the method through the result comparison with the well-known analytical solution. A larger reduction could have been easily obtained employing a higher ratio of the smoothing lengths.

Dirichlet pressure boundary conditions have been imposed at the crosssections $A$ and $B\left(p_{A}=2 \cdot 10^{-3} P a\right.$ and $\left.p_{B}=0\right)$, as discussed by Monteleone et al. (2017), to obtain the selected pressure gradient, while adherence boundary conditions have been set on the external lateral surface of block 1 .

The velocity profiles across the pipe diameter at two intermediate time levels and at the steady-state are plotted in Fig. 9, showing a very good agreement with the analytical solution (Szymanski, 1932) and a quite satisfactory matching of the solutions near the block interface.

The time evolution of the effective particle numbers $N_{1}(t)$ and $N_{2}(t)$ is shown in Fig. 10, made non-dimensional with the relative starting numbers. The changes in the total number of particles are quite limited in both the blocks, with ratios $N_{1}(t) / N 0_{1}$ and $N_{2}(t) / N 0_{2}$ much lower than $0,1 \%$ in most of the computational time. Higher values (lower than 1\%) can be seen only at the 


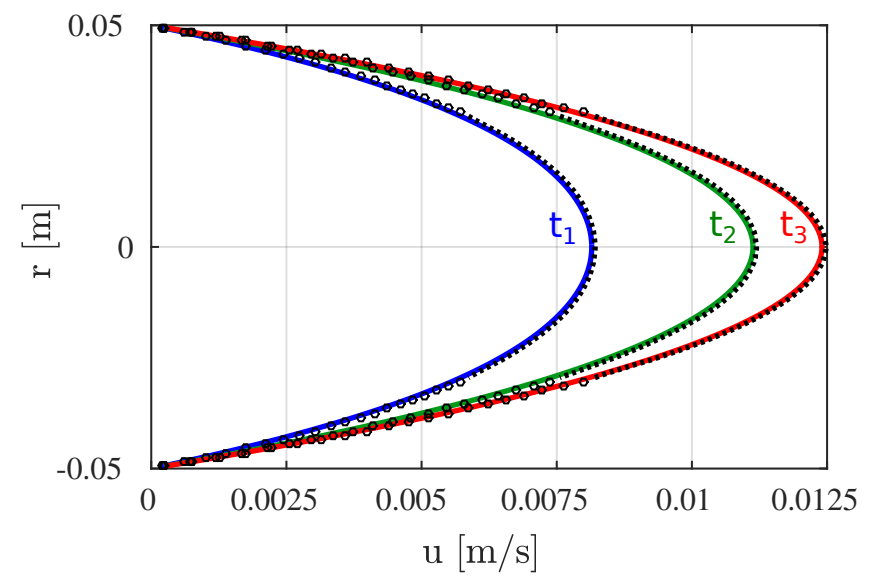

Figure 9: Test case 1. Velocity profile as a function of the radial coordinate $r$. Open circles: SPH solution in block 1. Dotted lines: SPH solution in block 2. Blue, green and red lines: analytical solutions at $t_{1}=500 \mathrm{~s}, t_{2}=1000 \mathrm{~s}$ and at the steady-state, respectively.

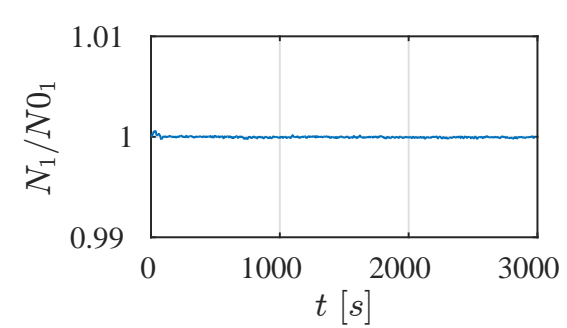

(a)

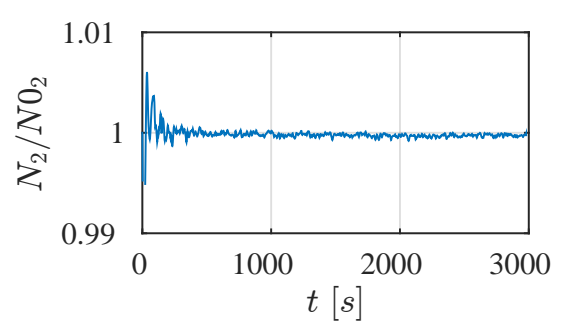

(b)

Figure 10: Test case 1. Time evolution of the numbers of particles $N_{1}(t)$ and $N_{2}(t)$ normalized with the initial numbers $N 0_{1}=62750$ and $N 0_{2}=10428$.

starting of the simulation, due to the perfectly regular initial distribution of the particles, which results in the leaving and entering of entire slices of particles through the inflow and outflow sections. This effect is particularly evident in block 2 where the particle velocity is larger, but is rapidly cancelled as the particle distribution becomes less regular.

\subsection{Test case 2: Von Kármán vortex shedding}

The 2D laminar flow around a circular cylinder has been analyzed, increasing the refinement of the solution in the vicinity of the immersed body. To this aim, three subdomains have been considered, as shown in Fig. 11, with blocks 2 and 3 in the annular regions close to the cylinder and block 1 elsewhere. The smallest value $k h_{3}=0.02 D$ (where $D=0.1 \mathrm{~m}$ is the diameter of the immersed cylinder) has been used in block 3 (between the diameters $D_{2}$ and $D$ ), the intermediate 


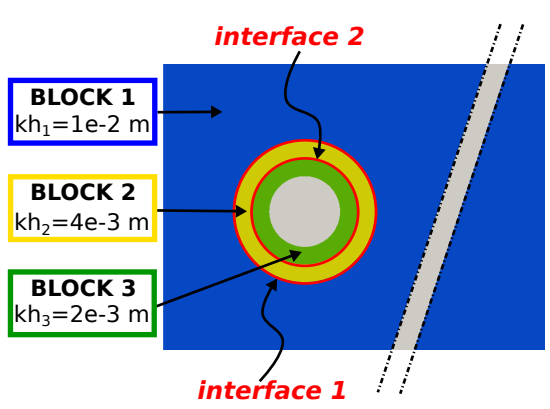

(a)

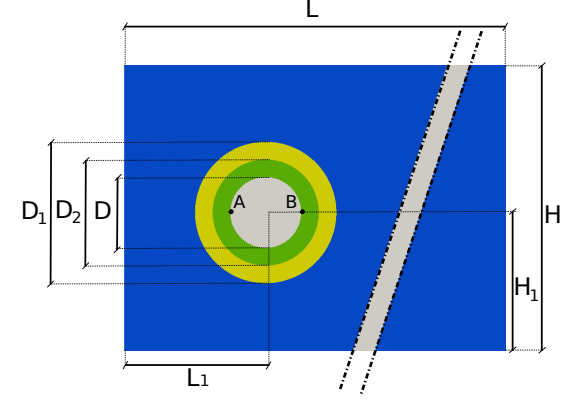

(b)

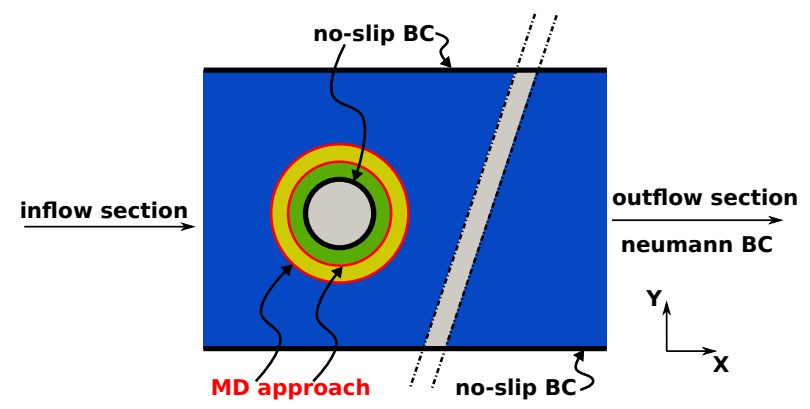

(c)

Figure 11: Test case 2. a) Domain subdivision into blocks 1 (blue), 2 (yellow) and 3 (green). The bold red lines indicate the two block interfaces; b) domain dimension. $L_{1}=0.2 \mathrm{~m}$, $L=2.2 \mathrm{~m}, D_{1}=0.2 \mathrm{~m}, D_{2}=0.15 \mathrm{~m}, D=0.1 \mathrm{~m}, H_{1}=0.20 \mathrm{~m}$ and $H=0.41 \mathrm{~m}$. Front point $A\left(x_{A}=0.15 m, y_{A}=0.20 m\right)$ and end point $B\left(x_{B}=0.25 m, y_{B}=0.20 m\right)$ of the cylinder; c) boundary conditions.

value $k h_{2}=0.04 D$ in block 2 (between $D_{2}=1.5 D$ and $D_{1}=2 D$ ) and the largest value $k h_{1}=0.1 D$ in block 1 , covering most of the domain. The resulting initial number of effective particles is 48086 in the whole domain (with 34816 particles in block 1, 3456 in block 2 and 9814 in block 3), about $5 \%$ of the value that would have been obtained using the smallest $k h(0.02 D)$ in the whole domain. During the simulation the number of particles in the blocks remained almost constant, with changes limited to $0.8 \%$ in the largest block 1 and $0.6 \%$ and $0.25 \%$ in blocks 2 and 3 , respectively.

The geometry, fluid properties and boundary conditions have been assigned as in the test case 2D-2 of Schäfer et al. (1996), which has been used for comparison. The length $L$ and the height $H$ of the computational domain have been set equal to $22 D$ and $4.1 D$, respectively. The centre of the cylinder is located at distance $L_{1}=2 D$ from the inflow section and $H_{1}=2 D$ from the bottom wall.

The fluid density and kinematic viscosity have been set to $\rho=1 \mathrm{~kg} / \mathrm{m}^{3}$ and $\nu=10^{-3} \mathrm{~m}^{2} / \mathrm{s}$, and a parabolic profile has been imposed at the inflow with mean velocity $u_{m}=1 \mathrm{~m} / \mathrm{s}$, resulting in the Reynolds number $R e=u_{m} D / \nu=$ 100. Since the simulation has been started from the rest, in order to obtain a 
smoother transition, the selected inflow velocity has been imposed after $0.1 s$ from the starting of the simulation, with a linear increase from the initial null value. Adherence boundary conditions have been used at the lateral walls and on the immersed body, while null velocity derivatives and null pressure have been imposed at the outflow.

The periodic detachment of vortices from either sides of the cylinder, characteristic of the considered flow at $R e$ values in the range of about $50 \div 150$, is easily identified in Figs. 12 and 13. Specifically, in Fig. 12.a the particle streamwise velocity is plotted at time $t=8 \mathrm{~s}$ after the starting of the simula-
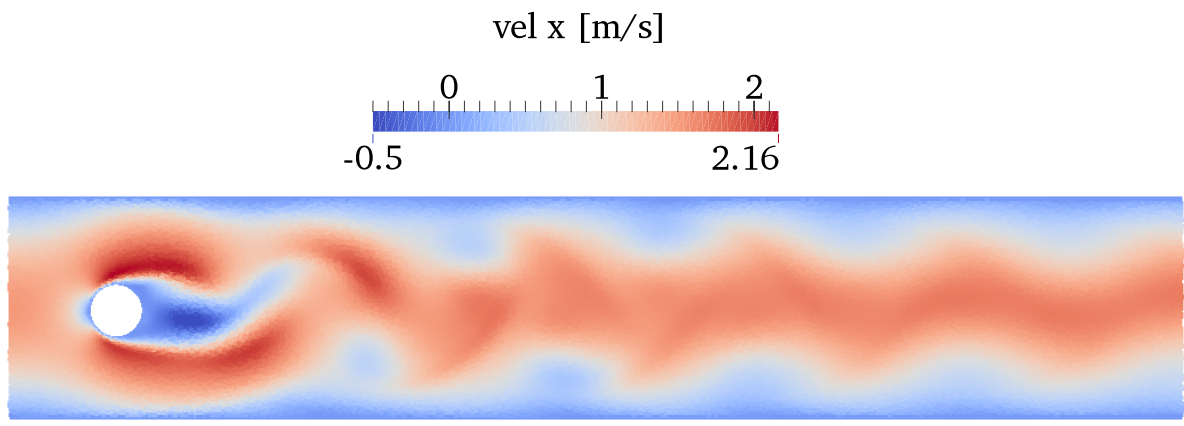

(a)

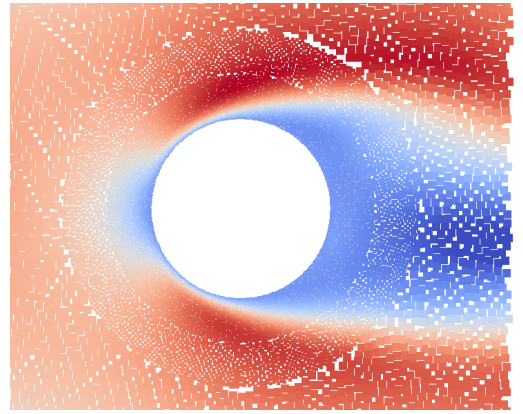

(b)

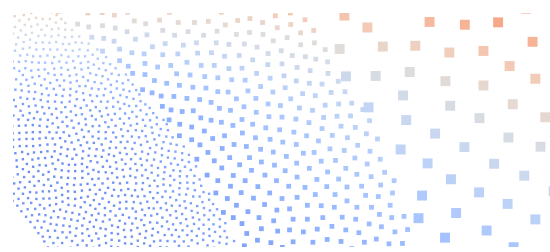

(d)

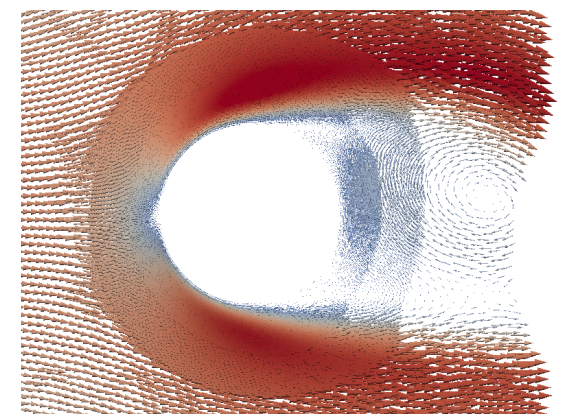

(c)

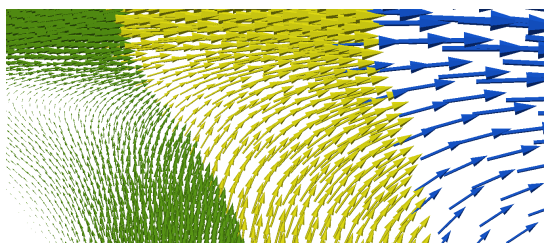

(e)

Figure 12: Test case 2. Velocity field at time $t=8 \mathrm{~s}$. a) Streamwise particle velocity in the whole domain; b) streamwise particle velocity near the circular cylinder; c) velocity vectors near the circular cylinder; d) particle distribution at the subdomain transitions; e) velocity vectors at the subdomain transitions colored as the corresponding blocks in figure 11. 


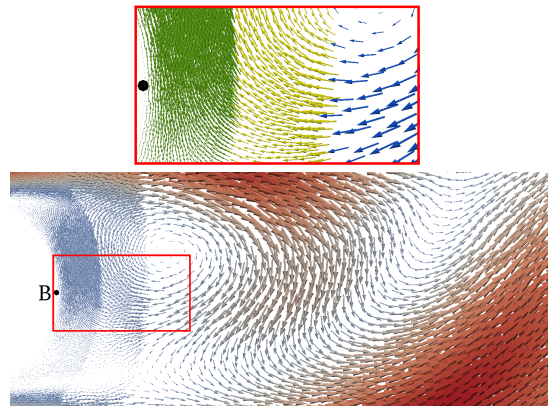

(a)

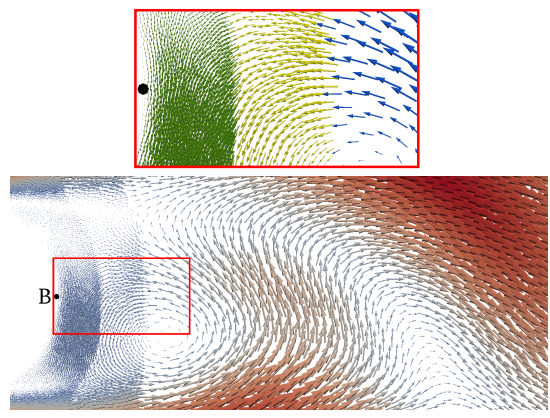

(c)

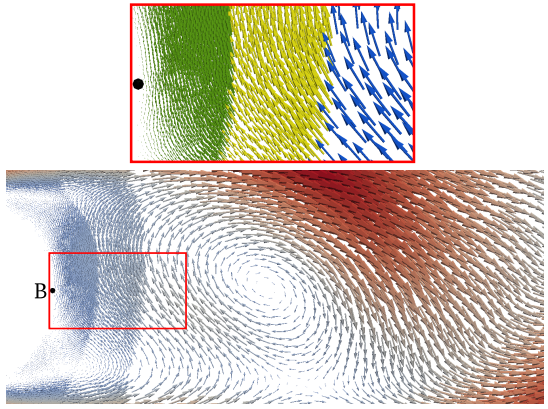

(b)

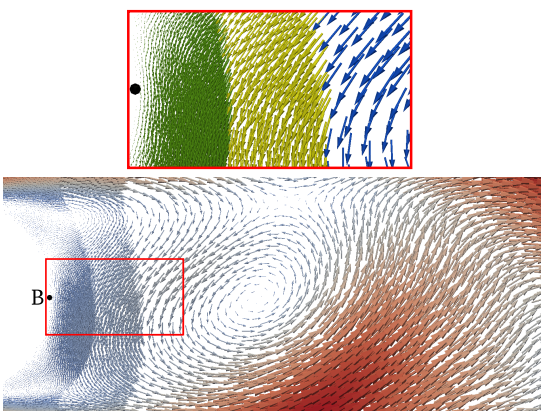

(d)

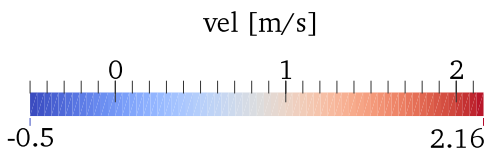

Figure 13: Test case 2. Velocity vectors at different times levels. a) $t=6.31 \mathrm{~s}$; b) $t+T / 4$; c) $t+T / 2 ; \mathrm{d}) t+3 / 4 T)$, where $T=0.328 s$ is the vortex shedding period. Enlargements inside the red lines: velocity vectors colored as in Fig. 11.

tion, corresponding to about 24 vortex shedding periods $T$ as it will be discussed below. An enlargement of the near cylinder region is shown in Figs. 12.b and 12.c, where the particle streamwise velocity and velocity vectors are plotted respectively, showing a very good matching of the solution through the block interfaces. A further enlargement is shown in Figs. 12.d and 12.e, which allow to clearly identify the increasing particle distance while moving outwards from the cylinder and the corresponding velocity vectors.

The vortices are shown in Fig. 13 at the time levels $t=6.31 \mathrm{~s}$ (one of the peaks of the lift coefficient that will be defined below), $t+T / 4, t+T / 2$ and $t+3 / 4 T$, where the enlargements inside the red rectangles highlight the vector transitions near the interfaces.

The smoke lines are plotted in Fig. 14 with reference to the inflow positions corresponding to the cylinder height (between $y=H_{1}-D / 2$ and $y=H_{1}+D / 2$ ), using different colors from red to light blue to indicate growing distances from the axis of the domain. 


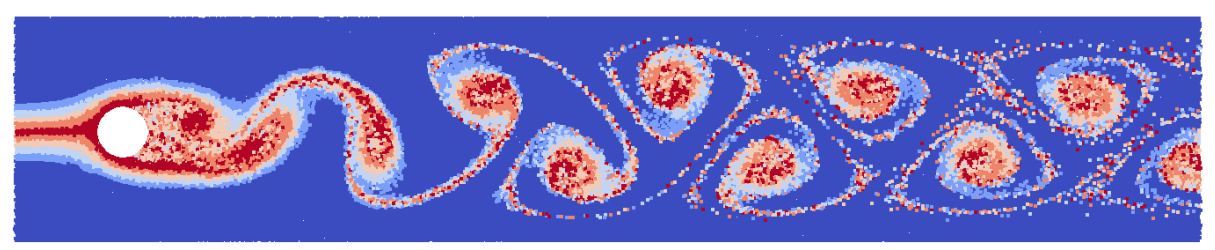

Figure 14: Test case 2. Smoke lines at time $t=8 \mathrm{~s}$.

The non-dimensional drag and lift coefficients have been calculated as

$$
\begin{aligned}
C_{D} & =\frac{2}{\rho u_{m}^{2} D} \int_{S}\left(\rho \nu \frac{\partial u_{t}}{\partial n} n_{y}-p n_{x}\right) d S \\
C_{L} & =-\frac{2}{\rho u_{m}^{2} D} \int_{S}\left(\rho \nu \frac{\partial u_{t}}{\partial n} n_{x}+p n_{y}\right) d S
\end{aligned}
$$

where $S$ is the cylinder surface (discretized into line segments in the $2 \mathrm{D}$ approximation), $u_{t}$ is the tangential velocity, $p$ is the pressure and $n$ is the surface normal direction pointing outwards, with components $n_{x}$ and $n_{y}$ in the horizontal and vertical directions respectively. The surface integrals in eq. (12) have been calculated in discrete form considering the middle points of the line segments. The time evolution of $C_{D}$ and $C_{L}$ is plotted in Figs. 15.a and 15.b which show that stable conditions, corresponding to the complete vortex devel-

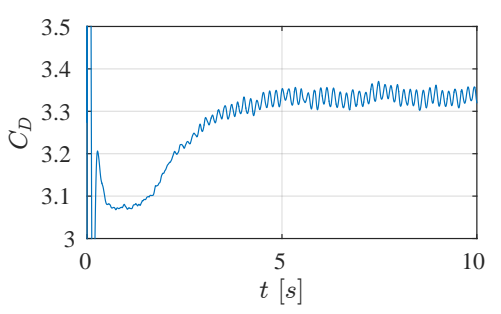

(a)

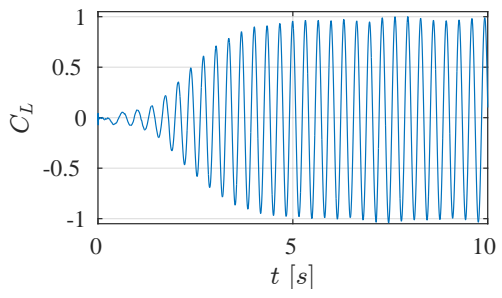

(b)

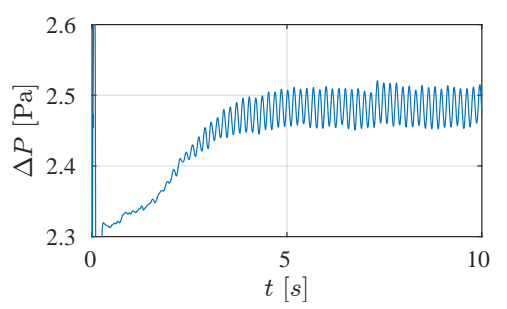

(c)

Figure 15: Test case 2. a) Drag coefficient $C_{D}$; b) lift coefficient $C_{L}$; c) pressure difference $\Delta p\left(p_{A}-p_{B}\right)$. 
opment, are achieved after about $5 \mathrm{~s}$. The maximum value of the drag coefficient after $t=5 \mathrm{~s}$ oscillates between 3.345 and 3.370, with an average value of 3.354, slightly larger (about 3.5\%) than the optimal value of $3.22-3.24$ estimated by Schäfer et al. (1996). Correspondingly, the maximum value of $C_{L}$ oscillates between 0.956 and 0.989 , with an average value of 0.972 , only $1.7 \%$ lower than the estimated optimal value of 0.99 .

The pressure difference between the front point $A\left(L_{1}-D / 2, H_{1}\right)$ and the end point $B\left(L_{1}+D / 2, H_{1}\right)$ of the cylinder (see Fig. 11.b) is plotted in Fig. 15.c. The obtained mean value in the time period $t=5 \div 10 \mathrm{~s}$ is $\Delta p=2.484 \mathrm{~Pa}$, in perfect agreement with the values suggested by Schäfer et al. (1996), where the range $2.46 \div 2.50 \mathrm{~Pa}$ is reported. The mean frequency of separation $f$ has been estimated from the period of oscillation $T$ of the lift coefficient $C_{L}$, resulting in the value $f=3.043 \mathrm{~Hz}$. Correspondingly, the value of the Strouhal number $S t=D f / u_{m}=0.304$ has been obtained, which is again in perfect agreement with the reference values of $0.295 \div 0.305$.

In order to show the accuracy of the proposed decomposition method, the comparison of the results with those obtained using a constant value of the smoothing length is shown in Fig. 16. The comparison is done with the results obtained at $t=2 \mathrm{~s}$ with $k h=0.04 D$. The velocity field and the smoke lines are plotted in figures 16.a and 16.b, respectively, showing a very good agreement with the one domain results. The pressure field in the vicinity of the cylinder is plotted in Fig. 16.c, showing again that the proposed multi-domain technique allows to obtain accurate results with a relevant reduction of the particle number.

The speed up of the proposed method is directly related to the reduction of the particle number since the overload of the computation due to the interface treatment is negligible. Specifically, the solution matching at the interfaces described in subsection 3.2, which is done twice at each time step (one after the predictor step and one after the velocity correction), requires a computational time lower than $1 \%$ of the entire iteration time. The particle generation at the interface (subsection 3.3) involves a computational time of about $0.7 \%$ of the entire time step. On the whole, thus, the total overload of the computational time due to the proposed multi-domain technique is limited to about $2.5 \%$ of the remaining computation.

\subsection{Test case 3: Blood flow analysis of a cerebral aneurysm}

In the third test case the simulation of the blood flow inside a human cerebral vessel with small branches and a giant aneurysm (Morley and Barr, 1969) is analysed. The geometry has been selected among the ones available at the AneuRisk project database (Aneurisk-Team, 2012), where the data of the vessel lateral surface discretized into triangles are provided (case C0093 of the available list). The domain, which has been used in section 3.1 (Fig. 2) to describe the partitioning into non-overlapping blocks, represents a high complex 3D geometry with branches having very different mean diameters and a quite large aneurysmal sac. A multi-resolution approach is thus necessary since the smoothing length $h$ in each branch should be at least 20 times smaller than the 


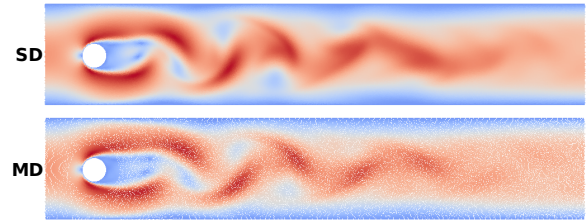

(a)

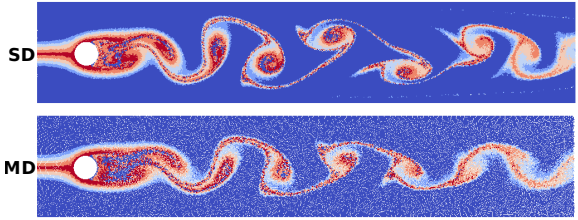

(b)

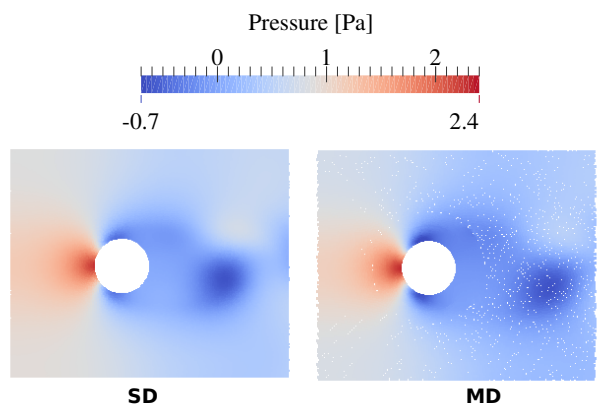

(c)

Figure 16: Test case 2. Comparison between single domain $(S D)$ and the proposed multidomain $(M D)$ approaches at $t=2 \mathrm{~s}$. a) Streamwise particle velocity. The scale is the same of Fig. 12 ; b) smoke lines; c) pressure field in the vicinity of the immersed body.

mean diameter to obtain a sufficiently accurate description of the velocity profile. As seen in Fig. 2, the computational domain has been thus subdivided in six blocks, having different values of $k h$ in the range $1 \div 5 \cdot 10^{-4} \mathrm{~m}$. Specifically, a quite small value of $k h$ was required in block $6\left(k h_{6}=1 \cdot 10^{-4} \mathrm{~m}\right)$, which would have been excessively small for the branches of blocks 1 and 5 and even more for the blocks 2 and 4 corresponding to the aneurysmal neck and sac, respectively. Thus, adopting a constant $k h$ in the whole domain would have implied a huge number of particles, with a resolution exceedingly high in most of the domain. Moreover, the pathological dilatation is characterized by relatively low velocities, making even less necessary the fine discretization.

The resulting total number of particles is 112618 , about $2 \%$ of the number that would have been obtained using the smallest $k h$ value $\left(1 \cdot 10^{-4} \mathrm{~m}\right)$ in the whole domain.

The boundary conditions for each block are shown in Fig. 17. At the inlet section $A$ pulsatile flow condition has been prescribed. To this aim a typical waveform of healthy individual has been chosen (Radaelli et al., 2008; Marzo et al., 2011), with period $T=0.792 s$ and time-averaged flow rate of about $0.3 \mathrm{ml} / \mathrm{s}$ obtained following the power law relationship between the flow rate and the cross-sectional area proposed by Cebral et al. (2008). The velocity profile at the inlet has been thus obtained applying the Womersley solution (Womersley, 1955). A constant pressure has been imposed at the outlet sections ( $B, C$ and $D$ in figure), while at the five block interfaces (red areas in the figure) the procedure described in sections 3.2 and 3.3 has been applied. 


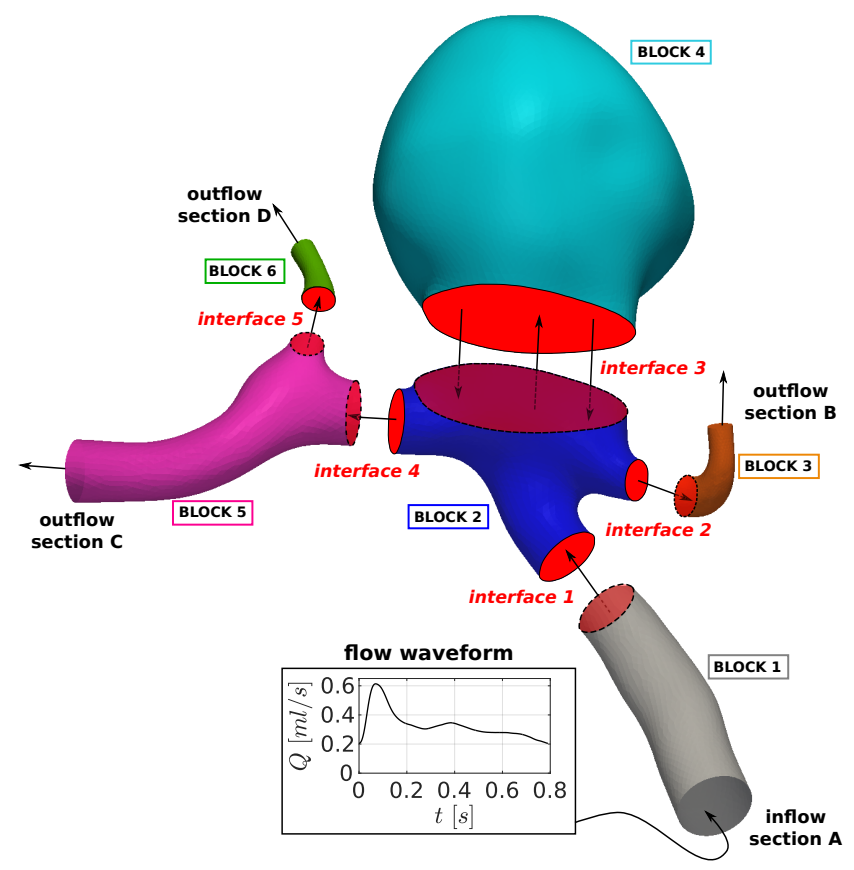

Figure 17: Test case 3. Boundary conditions for each block.

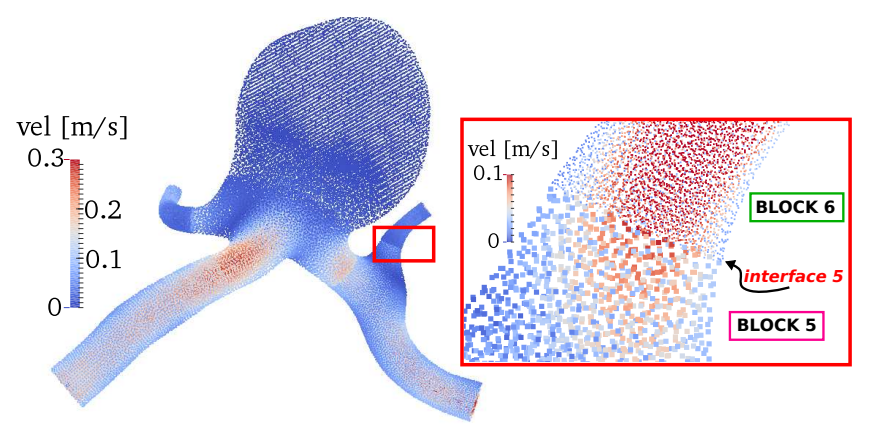

Figure 18: Test case 3. Particle velocity magnitude in $\mathrm{m} / \mathrm{s}$. Enlargement at interface 5 (red rectangle) separating block 5 from block 6 . 

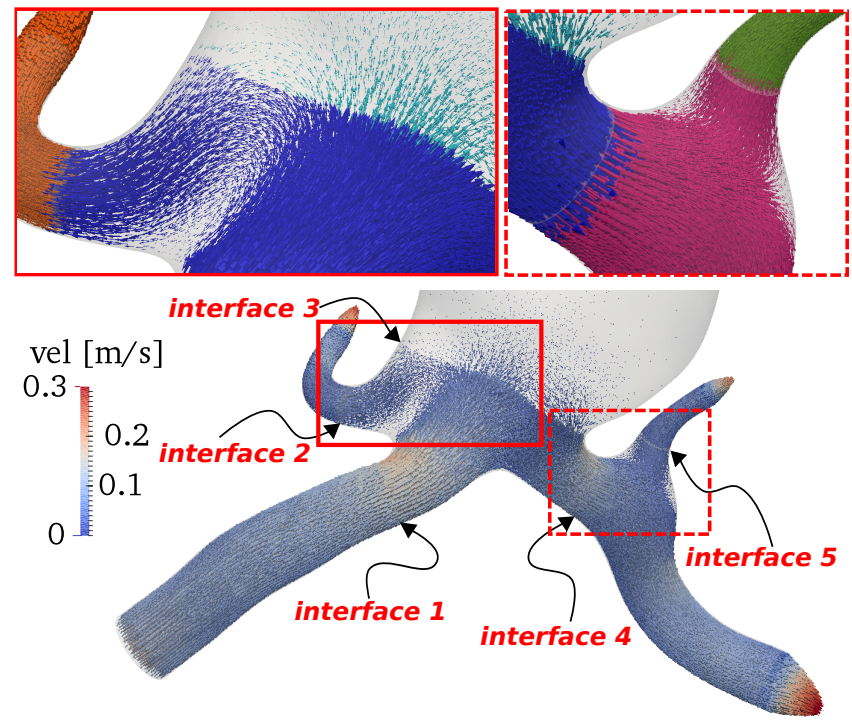

Figure 19: Test case 3. Velocity vectors. Enlargements at interfaces 2 and 3 (bold red line) and interfaces 4 and 5 (dashed red line) using different colors to indicate velocity vectors relative to particles belonging to different blocks (see scheme in Fig. 2 for the colors).

Rigid vessel walls have been considered with no-slip boundary conditions. For simplicity, blood has been considered a Newtonian fluid with constant kinematic viscosity $\nu=3.7 \cdot 10^{-6} \mathrm{~m}^{2} / \mathrm{s}$ and density $\rho=1060 \mathrm{~kg} / \mathrm{m}^{3}$. The simulation has been performed over six systolic cycles.

The Fig. 18 shows the particle velocity magnitude in the whole domain and an enlargement in the vicinity of interface 5 which highlights the very good matching of the solution in the neighboring blocks (5 and 6). Further enlargements are shown in Fig. 19 where the velocity vectors are plotted.

The pressure evolution in eight points distributed in the blocks along the vessel centerlines are shown in Fig. 20 during one systolic period. The plotted patterns are coherent with the imposed velocity flux at the domain inflow (where the point $P 1$ is placed) and show a correct time evolution of the pressure values from one block to the others, with correct pressure drops while moving in the downstream direction along the centerlines.

In this test case no comparison data are available, but it is very suitable to show the global efficiency of the proposed method, with an even larger reduction of the particle numbers with respect to the previous case discussed in section 4.2 , and the perfect satisfaction of the mass conservation. To this aim the volume discharges in different branch cross-sections have been calculated based on the number of particles going through the corresponding sections during a fixed amount of time $(\Delta t=0.002 \mathrm{~s})$. The obtained discharge $Q_{1}$ in the cross-section $S_{1}$ of the inflow vessel (Fig. 21.a) is thus compared with the sum of the discharges $Q_{3}$ and $Q_{5}$ in two cross-sections of the downstream branches 3 and 5 ( $S_{3}$ 

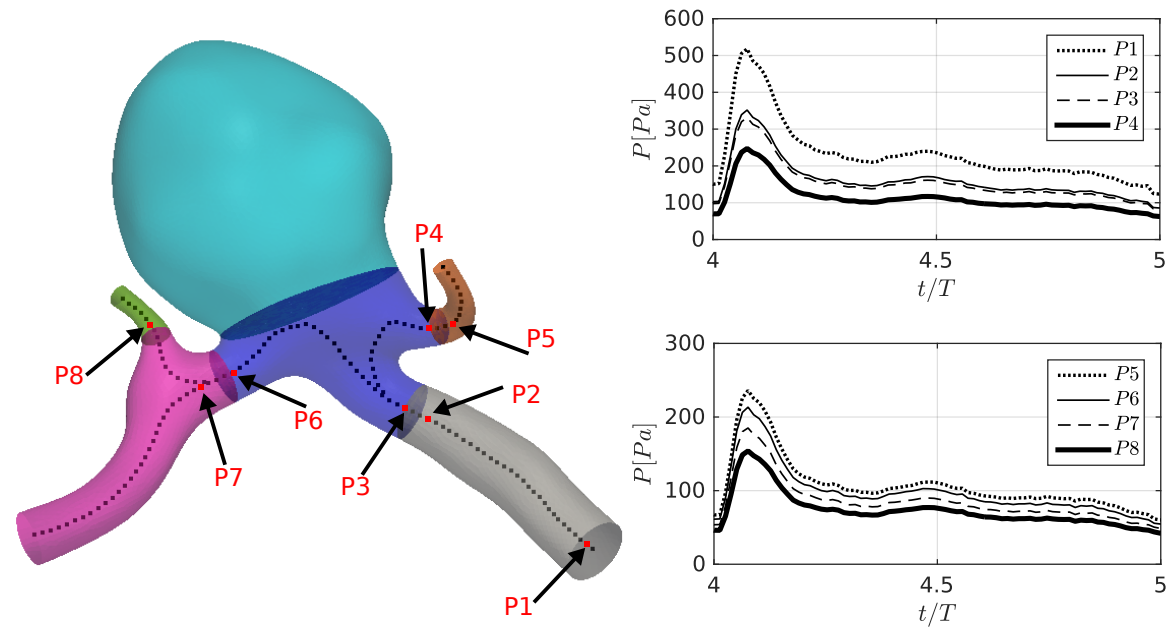

Figure 20: Test case 3. Pressure in $[\mathrm{Pa}]$ over one systolic cycle in different points of the centerline $(P 1, P 2, P 3, P 4, P 5, P 6, P 7, P 8)$.

and $S_{5}$ in the figure), showing that the continuity constraint is correctly obeyed $\left(Q_{1}=Q_{3}+Q_{5}\right)$. Since a further bifurcation occurs in block 5 downstream of the $S_{5}$ cross-section, the discharge $Q_{5}$ is compared in Fig. 21.b with the sum of the discharges in the downstream cross-sections $S_{5^{\prime}}$ (in the same block 5) and $S_{6}$ (in block 6), showing again that the mass conservation is fulfilled. It is worthwhile highlighting that the satisfaction of the continuity equation in cross-sections belonging to different subdomains in a Lagrangian method, although being a necessary requirement for a reliable solution, is not automatically guaranteed, since it is not explicitly enforced in the solved equations.

A further confirmation of the mass conservation satisfaction is obtained in Fig. 22 showing the time evolutions of the particle number $N_{n}(n=1, \ldots, 6)$ in each of the six blocks normalized with the corresponding initial number of particles $N 0_{n}\left(N 0_{1}=18241, N 0_{2}=17336, N 0_{3}=5735, N 0_{4}=30160\right.$, $\mathrm{NO}_{5}=32207$ and $\mathrm{NO}_{6}=9033$ ). The maximum amplitude of the oscillations in each block is always lower then $0.1 \%$ with the exception of the initial stages of the first systolic cycle where values of about $0.2 \%$ have been achieved. This result confirms the effectiveness of the proposed dynamic adjustment of the cone angle amplitude $\beta$ at the inlet interface of each block discussed in section 3.3.

\section{Concluding remarks}

The proposed method allows to alleviate one of the main drawbacks of the SPH method, which is the high computational cost with respect to mesh-based methods. This problem is not exclusively related to the method of discretization of the differential equations in itself, since it mostly depends on the difficulty to 
change the discretization refinement throughout the computational domain, a task which on the contrary is easily achieved in traditional grid methods. Thus, the employment of multi-domain techniques is one of the simplest strategy to overcome this issue in Lagrangian particle methods.

The proposed procedure can be straightforwardly implemented in the standard SPH formulation, managing the internal interfaces similarly to the external boundaries where usually mirror particles are generated to account for the support domain truncation and to apply the selected boundary conditions. Since no particle splitting/coalescing method is employed to take into account the variable kernel width when the particles move from one block to another, the procedure requires the simple deactivation of the particles leaving one block and, on the other side, the generation of new particles with the required size and mass in the receiving block. The release of new particles is governed by a search procedure of the existing particles in the vicinity of the block interfaces, using conical scan regions of assigned opening angle. In order to guarantee the conservation of mass, the frequency of release of new particles is increased or reduced to control the global fluid mass by dynamically adapting the cone opening angle.

The employed test cases show that, even in relatively long-term simulations of unsteady flows, the proposed procedure allows to conserve the global mass
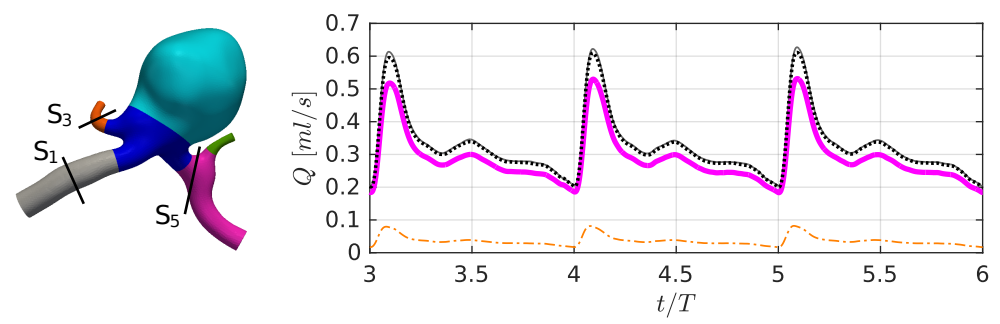

(a)
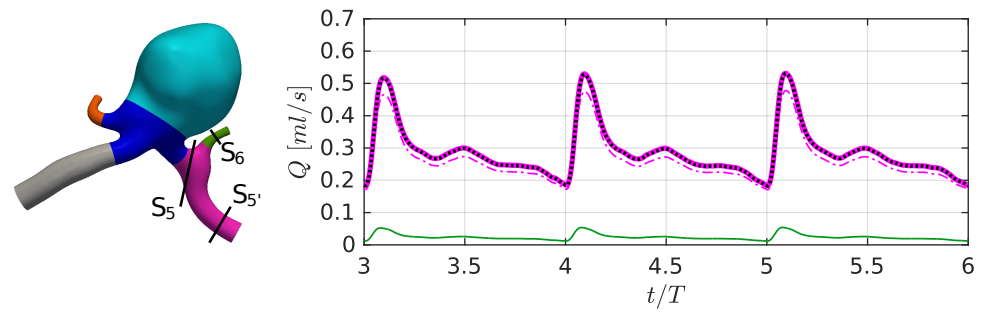

(b)

Figure 21: Test case 3. Flow rates $Q(t)$ in $\mathrm{ml} / \mathrm{s}$ over the last three cycles through the sections $S_{1}, S_{3}, S_{5}, S_{5^{\prime}}$ and $S_{6}$ (bold black lines in the domain sketch). a) Thin grey line: $Q_{1}$; dash-dot orange line: $Q_{3}$; magenta bold line: $Q_{5}$; dotted black line: $Q_{3}+Q_{5}$; b) dash-dot magenta line: $Q_{5^{\prime}}$; thin green line: $Q_{6}$; dotted black line: $Q_{6}+Q_{5^{\prime}}$. 


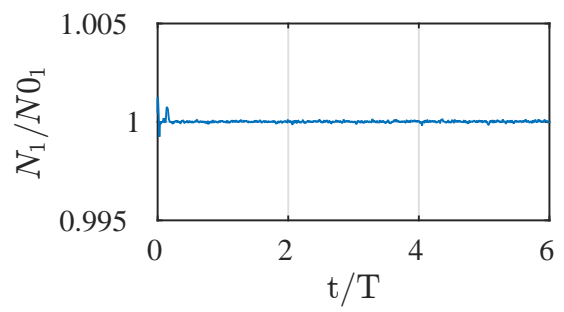

(a)

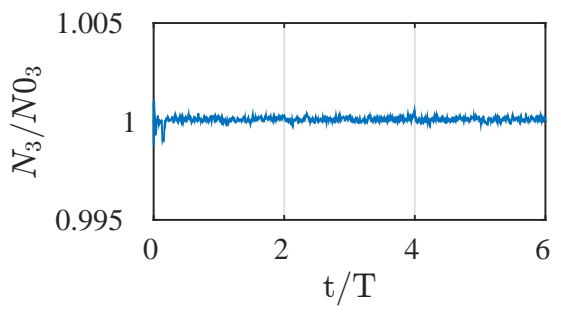

(c)

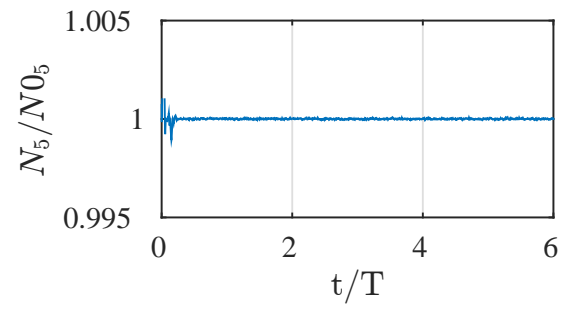

(e)

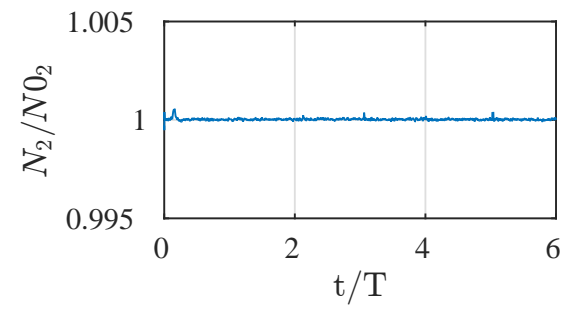

(b)

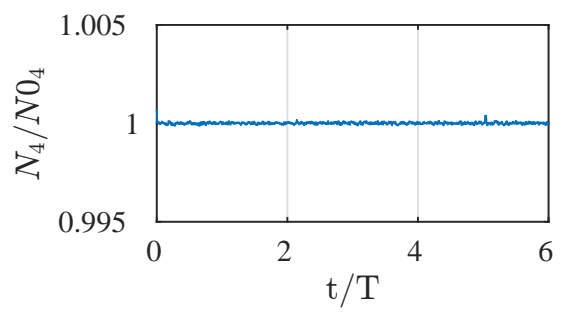

(d)

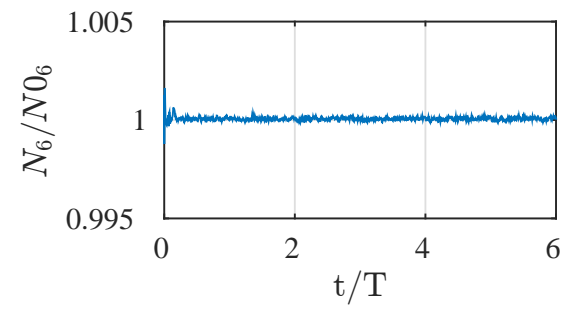

(f)

Figure 22: Test case 3. Time evolution of the number of particles over six systolic cycles. The time $t$ is normalized with the systolic period $T$ while the number of particles in each block with the starting number of particles $N 0_{n}$. a) block 1 ; b) block 2; c) block 3; d) block 4; e) block 5 ; f) block 6 . 
and that the mass discharges through cross-sections belonging to different blocks are in perfect agreement with the continuity equation. The numerical solutions are in very good agreement with the available comparison results, with seamless transition of the velocity fields through the interfaces between neighboring blocks.

The simulations have been performed employing values up to 2.5 of the ratio between the kernel widths of neighboring blocks (test case 2), and up to 5 between those of non-neighboring ones (e.g., blocks 4 and 6 of the test case 3 ). Since the number of particles in 2D and 3D SPH approximations is proportional to the second and third power of the kernel width, respectively, the multi-block approach allowed to obtain a very high reduction of the particle numbers (about 20 times in the $2 \mathrm{D}$ test case 2 and 50 times in the $3 \mathrm{D}$ test case 3 ). The resulting reduction of the computational time was almost proportional to that of the particle number since the overloads due to the interface management described in subsections 3.2 and 3.3 was almost negligible.

In principle even much higher reductions of the computational efforts could be achieved using the proposed multi-domain approach with higher values of the ratios of the employed smoothing lengths, thus extending the field of application of the SPH approach to real world fluid flow problems. As discussed in the Introduction, since the method can be in principle applied to free-surface flows, further validations will be performed in future research considering non-confined flows.

\section{References}

Aneurisk-Team, 2012. Aneurisk repository. Web Site.

URL http : //ecm2.mathcs.emory.edu/aneuriskweb

Barcarolo, D. A., Le Touzé, D., Oger, G., De Vuyst, F., 2014. Adaptive particle refinement and derefinement applied to the smoothed particle hydrodynamics method. Journal of Computational Physics 273, 640-657.

Cebral, J., Castro, M., Putman, C., Alperin, N., 2008. Flow-area relationship in internal carotid and vertebral arteries. Physiological measurement 29 (5), 585.

Feldman, J., Bonet, J., 2007. Dynamic refinement and boundary contact forces in sph with applications in fluid flow problems. International Journal for $\mathrm{Nu}-$ merical Methods in Engineering 72 (3), 295-324.

Hu, W., Pan, W., Rakhsha, M., Tian, Q., Hu, H., Negrut, D., 2017. A consistent multi-resolution smoothed particle hydrodynamics method. Computer Methods in Applied Mechanics and Engineering 324, 278 - 299.

Kunz, P., Hirschler, M., Huber, M., Nieken, U., 2016. Inflow/outflow with Dirichlet boundary conditions for pressure in ISPH. Journal of Computational Physics 326, 171-187. 
Lind, S., Xu, R., Stansby, P., Rogers, B. D., 2012. Incompressible smoothed particle hydrodynamics for free-surface flows: A generalised diffusion-based algorithm for stability and validations for impulsive flows and propagating waves. Journal of Computational Physics 231 (4), 1499-1523.

Liu, M., Liu, G., 2010. Smoothed particle hydrodynamics (SPH): an overview and recent developments. Archives of computational methods in engineering 17 (1), 25-76.

López, Y. R., Roose, D., Morfa, C. R., 2013. Dynamic particle refinement in sph: application to free surface flow and non-cohesive soil simulations. Computational Mechanics 51 (5), 731-741.

Marzo, A., Singh, P., Larrabide, I., Radaelli, A., Coley, S., Gwilliam, M., Wilkinson, I. D., Lawford, P., Reymond, P., Patel, U., et al., 2011. Computational hemodynamics in cerebral aneurysms: the effects of modeled versus measured boundary conditions. Annals of biomedical engineering 39 (2), 884-896.

Monaghan, J. J., 2000. Sph without a tensile instability. Journal of Computational Physics 159 (2), 290-311.

Monteleone, A., Monteforte, M., Napoli, E., 2017. Inflow/outflow pressure boundary conditions for smoothed particle hydrodynamics simulations of incompressible flows. Computers \& Fluids 159, 9-22.

Morley, T., Barr, H., 1969. Giant intracranial aneurysms: diagnosis, course, and management. Clinical neurosurgery 16, 73-94.

Morris, J. P., Fox, P. J., Zhu, Y., 1997. Modeling low Reynolds number incompressible flows using SPH. Journal of computational physics 136 (1), 214-226.

Napoli, E., 2011. Panormus user's manual. University of Palermo, Palermo, Italy, www.panormus_3d.org, 1-74.

Napoli, E., De Marchis, M., Gianguzzi, C., Milici, B., Monteleone, A., 2016. A coupled Finite Volume-Smoothed Particle Hydrodynamics method for incompressible flows. Computer Methods in Applied Mechanics and Engineering $310,674-693$.

Napoli, E., De Marchis, M., Vitanza, E., 2015. PANORMUS-SPH. A new Smoothed Particle Hydrodynamics solver for incompressible flows. Computers \& Fluids 106, 185-195.

Radaelli, A., Augsburger, L., Cebral, J., Ohta, M., Rüfenacht, D., Balossino, R., Benndorf, G., Hose, D., Marzo, A., Metcalfe, R., et al., 2008. Reproducibility of haemodynamical simulations in a subject-specific stented aneurysm modela report on the Virtual Intracranial Stenting Challenge 2007. Journal of biomechanics 41 (10), 2069-2081.

Saad, Y., 2003. Iterative methods for sparse linear systems. SIAM. 
Schäfer, M., Turek, S., Durst, F., Krause, E., Rannacher, R., 1996. Benchmark computations of laminar flow around a cylinder. In: Flow simulation with high-performance computers II. Springer, pp. 547-566.

Shibata, K., Koshizuka, S., Matsunaga, T., Masaie, I., 2017. The overlapping particle technique for multi-resolution simulation of particle methods. Computer Methods in Applied Mechanics and Engineering 325, 434-462.

Spreng, F., Schnabel, D., Mueller, A., Eberhard, P., 2014. A local adaptive discretization algorithm for smoothed particle hydrodynamics. Computational Particle Mechanics 1 (2), 131-145.

Szymanski, G., 1932. Quelques solutions exactes des équations d'hydrodynamique du fluide visqueux dans le cas d'un tube cylindrique. Journal de mathématiques pures et appliquées 11, 67-108.

Vacondio, R., Rogers, B., Stansby, P., Mignosa, P., 2013a. Shallow water sph for flooding with dynamic particle coalescing and splitting. Advances in water resources $58,10-23$.

Vacondio, R., Rogers, B., Stansby, P., Mignosa, P., 2016. Variable resolution for SPH in three dimensions: Towards optimal splitting and coalescing for dynamic adaptivity. Computer Methods in Applied Mechanics and Engineering 300, 442-460.

Vacondio, R., Rogers, B., Stansby, P., Mignosa, P., Feldman, J., 2013b. Variable resolution for SPH: a dynamic particle coalescing and splitting scheme. Computer Methods in Applied Mechanics and Engineering 256, 132-148.

Van der Vorst, H. A., 1992. Bi-CGSTAB: A fast and smoothly converging variant of Bi-CG for the solution of nonsymmetric linear systems. SIAM Journal on scientific and Statistical Computing 13 (2), 631-644.

Wendland, H., 1995. Piecewise polynomial, positive definite and compactly supported radial functions of minimal degree. Advances in computational Mathematics 4 (1), 389-396.

Womersley, J. R., 1955. Method for the calculation of velocity, rate of flow and viscous drag in arteries when the pressure gradient is known. The Journal of physiology 127 (3), 553-563.

Xiong, Q., Li, B., Xu, J., 2013. GPU-accelerated adaptive particle splitting and merging in SPH. Computer Physics Communications 184 (7), 1701-1707.

Xu, R., Stansby, P., Laurence, D., 2009. Accuracy and stability in incompressible SPH (ISPH) based on the projection method and a new approach. Journal of Computational Physics 228 (18), 6703-6725.

Zang, Y., Street, R. L., Koseff, J. R., 1994. A non-staggered grid, fractional step method for time-dependent incompressible Navier-Stokes equations in curvilinear coordinates. Journal of Computational Physics 114 (1), 18-33. 